Imperial/TP/93-94/38

NI94004

hep-th/9406067

\title{
On exact solutions and singularities in string theory
}

\author{
Gary T. Horowitz*** \\ Isaac Newton Institute \\ Cambridge CB3 OEH, U.K. \\ and \\ A.A. Tseytlin ${ }^{\star \dagger}$ \\ Theoretical Physics Group, Blackett Laboratory \\ Imperial College, London SW' 2BZ, U.K.
}

\begin{abstract}
We construct two new classes of exact solutions to string theory which are not of the standard plane wave or gauged WZW type. Many of these solutions have curvature singularities. The first class includes the fundamental string solution, for which the string coupling vanishes near the singularity. This suggests that the singularity may not be removed by quantum corrections. The second class consists of hybrids of plane wave and gauged WZW solutions. We discuss a four dimensional example in detail.
\end{abstract}

June 1994

* e-mail address: gary@cosmic.physics.ucsb.edu

** On leave from Physics Department, University of California, Santa Barbara, CA 93106, USA

* e-mail address: tseytlin@ic.ac.uk

$\dagger$ On leave from Lebedev Physics Institute, Moscow, Russia. 


\section{Introduction}

One of the main obstacles toward a better understanding of string theory is the scarcity of exact classical solutions. At the present time, only two classes of solutions are known for the bosonic string. The first are plane-wave-type backgrounds [1, 2, 3, [4] which have a covariantly constant null vector, and the second are those corresponding to gauged WZW models (see e.g. [5,60). (For the superstring, there is a third class of solutions corresponding to $(2,2)$ supersymmetric models.)

There is a well known ambiguity in the form of the classical string equations of motion. These equations are usually expressed as a power series in $\alpha^{\prime}$. The leading term is unambiguous, but the form of the higher order terms can be altered by field redefinitions or equivalently, by choosing different 'renormalization schemes'. For the simplest plane wave solutions this ambiguity is irrelevant since all the higher order terms vanish identically. For the gauged WZW solutions, in the familiar conformal field theory (CFT) scheme there are $\alpha^{\prime}$-corrections to all orders [7,8,9], but there is evidence [10,11, 12] that there also exists a scheme where the leading-order solution is exact. Given this ambiguity, to study string propagation and scattering one needs to know more than the fact that a particular background is exact in a certain scheme. One also needs to identify explicitly the corresponding CFT. This is known for the gauged WZW models, but not for all the plane wave solutions. Nevertheless, some properties of a solution can be determined from the information about its exact form in some scheme.

Is it possible to go beyond these two classes of solutions? Consider a family of backgrounds described by a dilaton $\phi(x)$ and a metric and antisymmetric tensor characterized by a single function $F(x)$ :

$$
d s^{2}=F(x) d u d v+d x_{i} d x^{i}, \quad B_{u v}=\frac{1}{2} F(x) .
$$

Note that the two functions $F$ and $\phi$ depend only on the transverse coordinates $x^{i}$. For backgrounds of this form, the leading order equations of motion reduce to (see Appendix A)

$$
\partial^{2} F^{-1}=2 b^{i} \partial_{i} F^{-1}, \quad \phi=\phi_{0}+b_{i} x^{i}+\frac{1}{2} \ln F(x),
$$

where $b_{i}$ is a constant vector. Some of the solutions to (1.2) have recently been shown to correspond to gauged WZW models where the subgroup being gauged is nilpotent [13]. It was argued that they should not receive higher order corrections in the CFT scheme. It is currently unknown whether all solutions to (1.2) can be obtained from a gauged WZW 
model but we believe this to be unlikely. Nevertheless, we will show that there is a scheme in which all of these solutions are exact and receive no $\alpha^{\prime}$ corrections. Since the equation for $F^{-1}$ is linear, linear combinations of these solutions yield new exact solutions.

One of the most interesting solutions in this class is the fundamental string (FS) [14] which has $b_{i}=0$ and

$$
\begin{gathered}
F^{-1}=1+\frac{M}{r^{D-4}}, \quad D>4 ; \\
F^{-1}=1-M \ln r, \quad D=4,
\end{gathered}
$$

where $r^{2}=x_{i} x^{i}$ and $D$ is the number of spacetime dimensions. This solution describes the field outside of a straight fundamental string located at $r=0$. The metric (1.1) becomes degenerate at $r=0$ and the curvature diverges. One would therefore expect the higher order terms in the string equations to become important and significantly modify the solution. However it was recently suggested [15] that this might not be the case for the superstring. Some evidence based on supersymmetry was presented that the FS does not receive higher order $\alpha^{\prime}$ corrections 1 One consequence of our results is that the FS is indeed an exact solution (in a particular scheme) even for the bosonic string theory, and thus of course is a heterotic or superstring solution as well.

It is known that the FS in $D>4$ is the extremal limit of a two parameter charged black string solution to the leading order equations with regular event horizon [16, 17]. The nonextremal black string receives higher order $\alpha^{\prime}$ corrections. But in $D=3$ a twoparameter charged black string solution was constructed from a gauged WZW model [18]. It is likely that there exists a scheme in which this leading order solution is exact (this was shown to order $\alpha^{\prime}$ in [11]). Its extremal limit turns out the be the general solution to (1.2) in three dimensions. This provides a perturbative check on the general argument for the conformal invariance of these backgrounds. In the original construction, the extremal $D=3$ black string was obtained by taking a certain limit of a background representing a gauged WZW model. We will show that this extremal solution can be obtained directly as a particular gauged $S L(2, R) \times R / R$ WZW model.

1 It was shown in [15] that corrections to the equations of motion coming from specific (anomaly-related) terms in the effective action vanish on the FS background. However, their argument is incomplete since the heterotic string effective action also contains other terms (necessary for reproducing the string $S$-matrix) which were not considered in 15. It may be that the contributions of these other terms (taken in a specific 'supersymmetric' scheme) also vanish on the FS background but this question deserves further investigation. 
The fact that the FS (1.3) is an exact classical solution has implications for singularities in string theory. We do not yet have a completely satisfactory definition of a singularity in classical string theory. Geodesic incompleteness, which is so useful in general relativity, is clearly unsatisfactory as seen e.g. by the example of orbifolds. Even diverging curvature is not a sufficient condition since some solutions with curvature singularities are known to be equivalent to nonsingular backgrounds. A definition based on the motion of classical strings is no better than geodesics, since null geodesics are included in the motion of strings. It appears that one must define a singularity in terms of the motion of quantum strings. This is natural since the equations for the background fields come from requiring that quantum strings are described by a CFT. Thus a string singularity should be a CFT which is ill-behaved in some sense. For the simplest plane wave solutions, one can study the propagation of quantum strings explicitly and show that this is not well behaved when the wave becomes singular [2] (see also [3]). So at least some classical singularities exist in string theory.

We do not yet know if the CFT associated with the FS is ill-behaved due to the curvature singularity. If it is, this may have a striking consequence. One usually expects that quantum effects will be large near regions of large curvature. But the string coupling is a dynamical field $e^{\phi}$, and for the FS solution $e^{\phi} \rightarrow 0$ near the singularity. This is quite different from other familiar examples of classical solutions such as the two dimensional black hole [5] for which $e^{\phi}$ diverges at the singularity in the leading order metric. (If one just considers solutions to the leading order equations, one can obtain a large class of singular backgrounds by starting with any regular solution with a symmetry having a fixed point, and applying a spacetime duality transformation [19]. In all these examples, $e^{\phi}$ diverges at the singularity.) The fact that $e^{\phi} \rightarrow 0$ for the FS suggests that quantum loop corrections will be suppressed and the solution will become more classical near the singularity. If this could be established, it would show that at least some singularities remain even in quantum string theory.

The observation that all the leading order solutions of the form (11.1) are exact also has implications for spacetime duality. These solutions can be obtained by applying a leading order duality transformation (with respect to translations in $u$ ) to the plane wave metrics

$$
d s^{2}=d u d v+K(x) d u^{2}+d x^{i} d x_{i},
$$

with $K=F^{-1}, B_{\mu \nu}=0$ and $\phi=\phi_{0}+b_{i} x^{i}$. These solutions are known to be exact. One can ask whether there always exists a scheme in which the leading order duality is not 
modified by $\alpha^{\prime}$-corrections. It turns out that this is not the case: the $\alpha^{\prime}$-modification [20] of the leading-order duality is necessary in all the schemes if $D \geq 3$. (A special scheme where duality is not modified exists in $D=2$; this is not surprising since as we shall see the effective action is trivial in this case.) However, it is possible that the following slightly weaker statement is true: Given an exact solution to string theory with a continuous symmetry, the solution obtained by a leading order duality transformation is also exact in some scheme.

This conjecture does not require that duality itself be exact since we allow the original solution and its dual to be exact in different schemes. Some earlier evidence for this came from the fact that the three dimensional black hole constructed from the $S L(2, R)$ WZW model [21] (which is exact) is dual to the three dimensional black string [18], and it was shown [11] that the first $\alpha^{\prime}$ correction to the black string metric can be removed by a field redefinition. The fact that the leading order duals to the plane wave solutions (1.4) also turn out to be exact is further support for this conjecture.

The method we will use to establish the conformal invariance of (1.1) also applies to a larger class of backgrounds where the transverse space is curved. A similar argument can be used to show the conformal invariance of the plane wave metrics (1.4) with non-flat transverse part (see also [4]). To obtain explicit solutions, one needs the 'transverse' theory to be, e.g., a gauged WZW model. In this way one obtains a 'hybrid' of the plane wave and gauged WZW solutions.

To illustrate this construction, we will discuss two examples. The smallest dimension for which the construction is nontrivial is four. In this case we are able to resolve a difficulty with the FS in four dimensions. The FS (1.3) in $D=4$ differs from its higher dimensional analogs in that it has an extra singularity at a non-zero value of $r$. We will find that the dual of the new solution we construct can be viewed as a fundamental string in $D=4$ which is asymptotically flat and has no additional singularities. We will also discuss a simple five dimensional example (which may also be interpreted as a $D=4$ heterotic string solution).

It is clear that when considering exact solutions, the scheme dependence of the equations of motion, or effective action (EA) that reproduces them, plays an important role. We shall show that in $D=2$ one can actually represent the $(G, B, \phi)$ part of the EA only by the leading-order term. In other words, all of the higher order $\alpha^{\prime}$-terms can be redefined away. In retrospect this is not surprising for two reasons. First, in $D=2$, the metricdilaton system has no dynamical degrees of freedom and the only propagating mode is a 
massless tachyon. So there are no real massive exchanges and hence no genuine $\alpha^{\prime}$-vertices in the EA. Second, in $D=2$ the most general leading-order classical solution is the 'black hole' [5,22]. This corresponds to the $S L(2, R) / U(1)$ coset and it is known that there exists a scheme [10] where the leading order solution is not modified by higher-order corrections.

In $D=3$ one cannot remove all the higher order terms since the $(G, B, \phi)$ system has one degree of freedom. However, we will see that it is possible to choose a scheme in which the higher order terms depend only on derivatives of the dilaton.

The plan of this paper is as follows. In Section 2 we introduce the general models we wish to consider, and derive the conditions under which they are conformally invariant to all orders in $\alpha^{\prime}$. In the next section, we use these results to explicitly construct two new solutions, one in four spacetime dimensions, and the other in five. Section 4 is devoted to a discussion of the field redefinition ambiguity and the structure of the effective action in low dimensions. In Section 5 we will show that solutions to (1.2) in $D=3$ correspond to a gauged WZW model. Section 6 discusses the relation between solutions (in particular, the ones corresponding to gauged WZW models) in different schemes. Some concluding remarks are made in Section 7. The appendices contain some technical details and a further generalization of our models.

\section{Path integral argument for conformal invariance}

\subsection{Basic models}

We wish to study strings propagating in the background (1.1) with a curved transverse space. This propagation is described by the following $\sigma$-model

$$
L_{F}=F(x) \partial u \bar{\partial} v+\left(G_{i j}+B_{i j}\right)(x) \partial x^{i} \bar{\partial} x^{j}+\alpha^{\prime} \mathcal{R} \phi(x)
$$

where $\mathcal{R}$ is related to the worldsheet metric $\gamma$ and its scalar curvature by $\mathcal{R} \equiv \frac{1}{4} \sqrt{\gamma} R^{(2)}$. We will refer to this model as the ' $F$-model'. We will also study the following generalization of the plane waves

$$
L_{K}=\partial u \bar{\partial} v+K(u, x) \partial u \bar{\partial} u+\left(G_{i j}+B_{i j}\right)(x) \partial x^{i} \bar{\partial} x^{j}+\alpha^{\prime} \mathcal{R} \phi(u, x)
$$

which we will call the ' $K$-model'. When $K$ and $\phi$ are independent of $u$, these two models are simply related by leading order duality: the dual of (2.2) with respect to $u$ is (2.1) with $F=K^{-1}$. 
The $F$-model has a large symmetry group. It is invariant under the three Poincare transformations in the $u, v$ plane. Moreover, it is invariant under the infinite-dimensional symmetry $u \rightarrow u+f(\tau+\sigma), v \rightarrow v+h(\tau-\sigma)$, i.e. it has two chiral currents. In general, the $K$-model has only one null Killing vector $l=\partial / \partial v$, but it is covariantly constant. The special case where $\phi$ depends only on $u$, and

$$
G_{i j}=\delta_{i j}, \quad B_{i j}=0, \quad K(u, x)=w_{i j}(u) x^{i} x^{j}
$$

actually has translation invariance in all transverse directions. It can be put into the form $L_{K}=\partial u \bar{\partial} v+\tilde{G}_{i j}(u) \partial x^{i} \bar{\partial} x^{j}$. The general $K$-model is also invariant under $v \rightarrow v+h(\tau-\sigma)$, i.e. it has one chiral current.

The fact that the $K$-model has a covariantly constant null vector can be used to give a simple geometrical argument that leading order solutions are exact in the special case when $G_{i j}=\delta_{i j}, B_{i j}=0$, and $\phi$ depends only on $u$ [2]. This is because the curvature contains two powers of the constant null vector $l$, and derivatives of $\phi$ are also proportional to $l$. One can thus show that all higher order terms in the equations of motion vanish identically. Only the leading order equations turn out to be nontrivial. Can one extend this argument to the case (2.2) when the transverse space is nontrivial? Clearly, the curvature of the transverse space can now appear at all orders of $\alpha^{\prime}$. Let us suppose that the transverse space is known to be an exact solution in some scheme. Then the model (2.2) will be conformal with $K=0$. But the curvature of the metric with $K \neq 0$ is equal to the curvature of the metric with $K=0$ plus a term of the form $(\nabla \nabla K) l l$. Unfortunately, this can result in nontrivial corrections to the equations of motion at each order of $\alpha^{\prime}$. These corrections will be linear in $K$, so one learns that the exact equation for $K$ will also be linear. But from this argument, one cannot conclude that there is a scheme in which the leading order solution for $K$ is also exact. To establish this, one needs to explicitly study the conformal invariance conditions from the path integral which we now proceed to do.

\subsection{Generating functional and conformal invariance conditions}

We shall study the conditions of conformal invariance of the above models by directly looking at the path integral representation for the generating functional. To obtain the complete set of Weyl invariance equations we need to introduce sources for the $\sigma$-model fields and find out when the generating functional on a curved worldsheet does not depend on the conformal factor of the worldsheet metric. This is equivalent to the condition of the vanishing of the trace of the stress-energy tensor operator. 
To avoid duplication, we will start by considering a more general $\sigma$-model which includes both the $F$-model and the $K$-model 2

$$
L_{F K}=F(x) \partial u \bar{\partial} v+K(x, u) \partial u \bar{\partial} u+\left(G_{i j}+B_{i j}\right)(x) \partial x^{i} \bar{\partial} x^{j}+\alpha^{\prime} \mathcal{R} \phi(u, x) .
$$

After proceeding as far as we can in this general theory, we will specialize to the two cases of interest, the $K$-model $(F=1)$ and the $F$-model $(K=0, \phi=\phi(x))$. 目 A slight generalization of the $F$-model which preserves its conformal invariance is discussed in Appendix B.

Let us define the generating functional

$$
\begin{gathered}
\exp (-W[U, V, X, \gamma])=\int[d u d v d x] \exp \left(-\frac{1}{\pi \alpha^{\prime}} \int d^{2} z\right. \\
\left.\left[L_{F K}(u, v, x, \gamma)+V \partial \bar{\partial} u+U \partial \bar{\partial} v+X \partial \bar{\partial} x\right]\right),
\end{gathered}
$$

where $U, V, X$ are external sources on the worldsheet with metric $\gamma_{p q}$ (which is taken in the conformal gauge). Since $v$ only appears linearly in the action, one can do the integral over it explicitly (by rotating $v \rightarrow i v$ ), obtaining a $\delta$-function factor

$$
\begin{gathered}
\exp (-W[U, V, X, \gamma])=\int[d u d x] \delta[\bar{\partial}(F(x) \partial u-\partial U)] \exp \left(-\frac{1}{\pi \alpha^{\prime}} \int d^{2} z\right. \\
\left.\left[K(x, u) \partial u \bar{\partial} u+V \partial \bar{\partial} u+\left(G_{i j}+B_{i j}\right)(x) \partial x^{i} \bar{\partial} x^{j}+\alpha^{\prime} \mathcal{R} \phi(u, x)+X \partial \bar{\partial} x\right]\right) .
\end{gathered}
$$

The $\delta$-function now allows us to do the integral over $u$ to obtain

$$
\begin{gathered}
\exp (-W[U, V, X, \gamma])=\int[d x] \Omega[x, \gamma] \exp \left(-\frac{1}{\pi \alpha^{\prime}} \int d^{2} z\right. \\
\left.\left[\left(G_{i j}+B_{i j}\right)(x) \partial x^{i} \bar{\partial} x^{j}+K\left(x, u_{*}\right) \partial u_{*} \bar{\partial} u_{*}+V \partial \bar{\partial} u_{*}+\alpha^{\prime} \mathcal{R} \phi\left(u_{*}, x\right)+X \partial \bar{\partial} x\right]\right),
\end{gathered}
$$

where $u_{*}$ is defined by ( $f$ is an arbitrary holomorphic function)

$$
F(x) \partial u_{*}=\partial U+f(z) \equiv \partial U^{\prime} .
$$

2 Low energy solutions of this form have been discussed in e.g. 223.

3 By adding a multiple of $u$ to $v$, one can shift $K$ by a multiple of $F$. So the theory (2.4) with $K=b F$ ( $b$ constant) is also equivalent to the $F$-model. Note also that in the case when $K$ does not depend on $u$, setting $u=y_{1}+y_{2}, v=y_{1}+q y_{2}$ and applying a duality transformation in $y_{2}$ direction we find $\left(u, v \rightarrow u^{\prime}, v^{\prime}\right) F^{\prime}=-F /(K+q F), \quad K^{\prime}=-1 /(K+q F)$. 
The determinant factor $\Omega$ is defined as follows. If the measure for the $(u, v)$ fields is given by

$$
<\delta u, \delta v>=\int d^{2} z \sqrt{\gamma} F_{0}(x) \delta u \delta v
$$

then

$$
\begin{gathered}
\Omega[x, \gamma]=(\operatorname{det} Q)^{-1} \equiv \int[d u d v] \exp \left[-\frac{1}{\pi \alpha^{\prime}} \int d^{2} z F(x) \partial u \bar{\partial} v\right] \\
=\int[d u d v] \exp \left[-\frac{1}{\pi \alpha^{\prime}}<u, Q v>\right], \\
Q \equiv-\frac{1}{\sqrt{\gamma} F_{0}} \partial(F \bar{\partial}) .
\end{gathered}
$$

The crucial point is that $\ln \operatorname{det} Q$ can be computed explicitly and has a local form. The general expression for $\operatorname{det} Q$ was found (in heat kernel regularisation) in 24 回

$$
\begin{gathered}
\Delta I \equiv-\ln \Omega=\frac{1}{8 \pi} \int d^{2} z \sqrt{\gamma} \Lambda^{2}\left(\ln F-\ln F_{0}\right)+\frac{1}{48 \pi} \int R^{(2)} \nabla^{-2} R^{(2)} \\
-\frac{1}{12 \pi} \int d^{2} z\left(\partial \ln F \bar{\partial} \ln F+\partial \ln F_{0} \bar{\partial} \ln F_{0}+4 \partial \ln F \bar{\partial} \ln F_{0}\right) \\
-\frac{1}{24 \pi} \int d^{2} z \sqrt{\gamma} R^{(2)}\left(2 \ln F+\ln F_{0}\right)
\end{gathered}
$$

where $\Lambda$ is an UV cutoff.

As usual, the form of $\ln \operatorname{det} Q$ in (2.12) is not unambiguous being dependent on a regularisation and choice of measure, i.e. is defined modulo local dimension 2 counterterms. What is unambiguous is the locality property of (2.12).

The definition of the determinant, i.e. the choice of $F_{0}$ and regularisation must be determined by the conditions on the whole $(x, u, v)$ theory (2.4). For example, if we use the heat kernel regularisation and demand target space covariance in the $(x, u, v)$ space we

4 A similar expression was also given in [25]. The reason why this determinant is given by a local expression can be understood in a simple way by drawing an analogy with a complex scalar coupled to a $U(1)$ gauge field: $L=(\partial \psi+i B \psi)\left(\bar{\partial} \psi^{*}-i \bar{B} \psi^{*}\right)$. If we set $u^{\prime}=\sqrt{F} u$ and $v^{\prime}=\sqrt{F} v$, then the Lagrangian in (2.10) takes the form $L=\left(\partial u^{\prime}+B u^{\prime}\right)\left(\bar{\partial} v^{\prime}-\bar{B} v^{\prime}\right), \quad B=-\frac{1}{2} \partial \ln F, \bar{B}=\frac{1}{2} \bar{\partial} \ln F$, so that in the present case the gauge field potential is purely transversal, $\mathcal{F}=\partial \bar{B}-\bar{\partial} B=\partial \bar{\partial} \ln F$. The logarithm of the determinant is proportional to that of a 2-dimensional Dirac fermion coupled to this gauge field, and the standard Schwinger-type term in the effective action $\sim \int \mathcal{F}(\partial \bar{\partial})^{-1} \mathcal{F}$ is equal to $\int \ln F \partial \bar{\partial} \ln F$. 
should set $F_{0}=F$. 5 This is because the covariant functional measure for a $\sigma$-model with the target space metric $G_{\mu \nu}$ is defined by $\left\langle\delta x, \delta x^{\prime}>=\int d^{2} z \sqrt{\gamma} G_{\mu \nu}(x) \delta x^{\mu} \delta x^{\prime \nu}\right.$. Then [24] (we do not indicate explicitly the free-theory $\gamma$-dependent term in (2.12))

$$
\Delta I=-\frac{1}{2 \pi} \int d^{2} z \partial \ln F \bar{\partial} \ln F-\frac{1}{8 \pi} \int d^{2} z \sqrt{\gamma} R^{(2)} \ln F
$$

or, equivalently,

$$
\Delta I=-\frac{1}{2 \pi} \int d^{2} z\left(\partial_{i} \ln F \partial_{j} \ln F \partial x^{i} \bar{\partial} x^{j}+\mathcal{R} \ln F\right) .
$$

The two terms in (2.14) lead to the local shifts of the metric $G_{i j}(x)$ and dilaton

$$
G_{i j}^{\prime}=G_{i j}-\alpha^{\prime} X_{i j}, \quad \phi^{\prime}=\phi-\frac{1}{2} \ln F, \quad X_{i j}=\frac{1}{2} \partial_{i} \ln F \partial_{j} \ln F
$$

It should be emphasized that the way we compute the path integral (2.5) (by directly integrating over $u, v)$ does not manifestly preserve the covariance in the $(x, u, v)$ space. One usually employs the normal coordinate expansion in order to maintain the covariance of perturbation theory. The use of 2.12 with $F_{0}=F$ in general is not sufficient to guarantee the target space covariance of the full theory.6 That means one may need to add extra local $\partial x \bar{\partial} x$ non-covariant counterterms in order to restore the target space covariance, i.e. $X_{i j}$ in (2.15) may contain extra local terms constructed out of derivatives of $\phi$ and $F$. Q

Returning to functional integral (2.7), we see that since $u_{*}$ is a non-local functional of $x$ the conditions of Weyl invariance of the resulting theory for $x^{i}$ are hard to determine in a closed form. We can, however, proceed further in our two special cases of interest:

$$
\text { (i) } F=1 \text { and } \quad \text { (ii) } K=0, \phi=\phi(x) \text {. }
$$

5 For generic choice of measure/regularisation one may need to make a non-covariant redefinition of the dilaton field $(\phi \rightarrow \phi+a \ln \operatorname{det} G$ ) in order to restore the target space covariance of the model 26].

6 The covariance in the transverse $x$-space is of course preserved under proper choice of regularisation/measure in the $x$-theory.

7 One may question why the addition of such counterterms is legitimate given that $\ln \operatorname{det} Q$ in (2.12) does not contain divergences of such kind. The point, however, is that extra divergences may be present in the general $(x, u, v)$ theory which should thus admit the corresponding freedom of local coupling redefinitions. 


\subsection{K-model}

In the $K$-model (2.2), $F=F_{0}=1$ so that the operator $Q$ (2.11) is trivial and thus $u_{*}=U(z)$ is $x$-independent (we absorb an arbitrary harmonic zero mode of $Q$ in $U$ ). The resulting path integral is

$$
\begin{gathered}
\exp (-W[U, V, X, \gamma])=Z_{0}(\gamma) \int[d x] \exp \left(-\frac{1}{\pi \alpha^{\prime}} \int d^{2} z\left[\left(G_{i j}+B_{i j}\right)(x) \partial x^{i} \bar{\partial} x^{j}\right.\right. \\
\left.\left.+T(x, U)+\alpha^{\prime} \mathcal{R} \phi(U, x)+X \partial \bar{\partial} x+V \partial \bar{\partial} U\right]\right) \\
T(x, U) \equiv K(x, U) \partial U \bar{\partial} U
\end{gathered}
$$

Now it is easy to formulate the conditions of the Weyl invariance of this theory: (1) the transverse $x$ - model $\left(G_{i j}, B_{i j}, \phi\right)$ must be Weyl invariant by itself (i.e., for $\left.U=0\right)$; $(2)$ since the interaction potential $T$ is equivalent (in what concerns its quantum field $x$ dependence) to a scalar 'tachyonic' term, it should solve the 'tachyonic' Weyl anomaly equation which is linear (to all orders in perturbation theory in $\alpha^{\prime}$ ) in $T$ [27]. Since $T$ is proportional to $K$ we get

$$
\begin{aligned}
& -\omega T+\partial^{i} \phi \partial_{i} T+2 \partial_{u}^{2} \phi \partial U \bar{\partial} U=0 \rightarrow \\
& -\frac{1}{2} \nabla^{2} K+O\left(\alpha^{\prime}\right)+\partial^{i} \phi \partial_{i} K+2 \partial_{u}^{2} \phi=0 .
\end{aligned}
$$

Here $\omega$ is the scalar anomalous dimension operator which in general contains $\left(G_{i j}, B_{i j}\right)$ dependent corrections to all orders in $\alpha^{\prime}$ and only a few leading $\alpha^{\prime n}$-terms in it are known explicitly (for a review see [10]).8 The dilatonic terms appear due to the $x$ and $U$-dependence of the dilaton.9 Note also that in contrast to the usual tachyonic coupling, here $T$ has canonical dimension 2 , so there is no ' -2 ' (tachyonic mass) term in this equation. The equation (2.18) can also be interpreted as the $u u$-component of the metric $\bar{\beta}$-function of the original $D$ dimensional sigma model (see [4]).

Given an exact string solution $\left(G_{i j}, B_{i j}, \phi\right)$, in general, we would still be unable to determine the exact expression for $K$ because of the unknown higher order terms in (2.18).

8 Note that we have assumed that the path integral is computed in the 'minimal subtraction' scheme where higher-order tadpoles do not produce contributions to the Weyl anomaly so that the operator $\omega$ does not contain higher-derivative terms in the flat space limit.

9 Computing the variation over the conformal factor of $\gamma$ one finds the 'classical' anomaly term $\sim \partial \bar{\partial} \phi$ which (after use of classical equations of motion) gives $\nabla_{i} \nabla_{j} \phi$ and $\nabla_{u} \nabla_{u} \phi=\partial_{u}^{2} \phi+$ $\frac{1}{2} G^{i j} \partial_{i} K \partial_{j} \phi$ in the relevant terms in the operator of the trace of the stress tensor. 
There are, however, special cases when this is possible. An obvious one is that of the flat transverse space with the dilaton being linear in the coordinate $x$

$$
G_{i j}=\delta_{i j}, \quad B_{i j}=0, \quad \phi=\phi_{0}(u)+b_{i}(u) x^{i}
$$

Then the exact equation for $K(2.18)$ becomes

$$
-\frac{1}{2} \partial^{i} \partial_{i} K+b^{i} \partial_{i} K+2 \partial_{u}^{2} \phi=0
$$

and can be readily solved. For $b_{i}=0$ we obtain the previously discussed plane wave type solutions [1,2, 3, 4]. The special case where

$$
K=1+\frac{M}{r^{D-4}}, \quad r^{2} \equiv x_{i} x^{i}, \quad \phi=\mathrm{const}
$$

is dual to the FS background [17] and describes a string boosted to the speed of light. For $b_{i} \neq 0$ one obtains a generalization of the plane wave type solutions with a linear dilaton.

We can obtain more interesting new exact solutions when the CFT behind the 'transverse' space solution $\left(G_{i j}, B_{i j}, \phi\right)$ is nontrivial but still known explicitly. In fact, in that case the structure of the 'tachyonic' operator $\omega$ is determined by the zero mode part of the CFT Hamiltonian, or $L_{0}$-operator. Fixing a particular scheme (e.g., the 'CFT' one where $L_{0}$ has the standard Klein-Gordon form with the dilaton term) we are then able, in principle, to establish the form of the background fields $\left(G_{i j}, B_{i j}, \phi\right)$ and $K$. This produces a hybrid of a gauged WZW and plane wave solution. Some examples in four and five dimensions will be discussed in Section 3.

\subsection{F-model}

Let us now turn to the second case when $K=0$ and $\phi=\phi(x)$. In that case the substitution of $u_{*}$ in (2.8) into the action in (2.7) gives

$$
\begin{gathered}
\exp (-W[U, V, X, \gamma])=\int[d x] \exp \left(-\frac{1}{\pi \alpha^{\prime}} \int d^{2} z\right. \\
\left.\left[\left(G_{i j}^{\prime}+B_{i j}\right)(x) \partial x^{i} \bar{\partial} x^{j}+T(x, U, V)+\alpha^{\prime} \mathcal{R} \phi^{\prime}(x)+X \partial \bar{\partial} x\right]\right) \\
T(x, U, V) \equiv-F^{-1}(x) \partial U^{\prime} \bar{\partial} V
\end{gathered}
$$

where we have used (2.14) and $G^{\prime}$ and $\phi^{\prime}$ were defined in (2.15). What we have obtained is a $\sigma$-model for $x^{i}$ with the 'massless' couplings $\left(G^{\prime}, B, \phi^{\prime}\right)$ and the 'tachyonic' coupling 
$T$. The dependence of $T$ on the background sources $U, V$ only implies that as in the $K$ model (2.16) and (2.17), $T$ has canonical dimension zero, not two. The condition of Weyl invariance is thus that $\left(G^{\prime}, B, \phi^{\prime}\right)$ should represent a Weyl-invariant theory and $T \sim F^{-1}$ should satisfy again eq.(2.18) (now with $\left(G^{\prime}, B, \phi^{\prime}\right)$ as background fields)

$$
-\omega^{\prime} T+\partial^{i} \phi^{\prime} \partial_{i} T=0 \rightarrow-\frac{1}{2} \nabla^{\prime 2} F^{-1}+O\left(\alpha^{\prime}\right)+\partial^{i} \phi^{\prime} \partial_{i} F^{-1}=0 .
$$

As mentioned above, this equation can be written down explicitly to all orders in $\alpha^{\prime}$ only when $\left(G^{\prime}, B, \phi^{\prime}\right)$ corresponds to a known CFT.

To summarize, given a conformal 'transverse' theory $\left(G^{\prime}, B^{\prime}, \phi^{\prime}\right)$ and $F$ satisfying (2.24), we find that for the particular choice of couplings in (2.15) the $F$-model with

$$
G_{i j}=G_{i j}^{\prime}+\frac{1}{2} \alpha^{\prime} \partial_{i} \ln F \partial_{j} \ln F, \quad \phi=\phi^{\prime}+\frac{1}{2} \ln F, \quad B_{i j}=B_{i j}^{\prime},
$$

represents an exact string solution (in a particular scheme the choice of which is implicit in the definition of the path integral we were discussing). Since the transverse theory is, in general, defined modulo local coupling redefinitions we may absorb the $\alpha^{\prime} \partial_{i} \ln F \partial_{j} \ln F$ term in (2.25) into a redefinition of $G_{i j}^{\prime}$ ( $F$ is an extra scalar from the point of view of the 'transverse' theory). We may also try to interpret this redefinition as a restriction of a field redefinition in the full $(u, v, x)$ theory.

As we have already mentioned above, the crucial point in our path integral argument is the locality of the relation between $G_{i j}$ and $G_{i j}^{\prime}$. The precise form of this relation would be fixed would we carry out the argument using some fixed explicit regularisation of the whole $(x, u, v)$ theory. If such a regularisation does not manifestly preserve the target space covariance we would need to make local non-covariant redefinitions of the $\sigma$ model couplings to restore the covariance in the final expressions.10 The locality of (2.15) is sufficient in order to be able to claim that there exists a scheme where the $F$-model represents an exact string solution. We can use the freedom of adding local non-covariant

10 Note that from the point of correspondence with field redefinitions in the effective action, the latter ones need not necessarily be covariant in order to preserve the $S$-matrix. The assumption of covariance is an extra condition that restricts the class of effective actions and field redefinitions one wishes to consider. From the quantum $2 d \sigma$-model point of view, target space covariance is an extra global symmetry in the space of $2 d$ fields and couplings that needs special effort (special choice of bare couplings) to be preserved in the full quantum $2 d$ theory. 
counterterms to $X_{i j}$ in (2.15) to put $G_{i j}-G_{i j}^{\prime}$ in a manifestly covariant backgroundindependent form which is the closest analog of $(2.25) 11$

$$
G_{\mu \nu}=G_{\mu \nu}^{\prime}+2 \alpha^{\prime} \partial_{\mu} \phi \partial_{\nu} \phi
$$

or simply to remove $X_{i j}$ completely,

$$
G_{\mu \nu}=G_{\mu \nu}^{\prime}
$$

This can then be considered as a transformation to a 'leading-order' scheme where the transverse metric is given just by the $\alpha^{\prime}$-independent $G_{i j}^{\prime}$.

Let us now consider some examples starting again with the simplest case:

$$
G_{i j}^{\prime}=\delta_{i j}, \quad B_{i j}=0, \quad \phi^{\prime}=\phi_{0}+b_{i} x^{i}, \quad \phi_{0}, b_{i}=\text { const }
$$

or, in terms of the original fields in the leading-order scheme (2.27)

$$
G_{i j}=\delta_{i j}, \quad \phi=\phi_{0}+b_{i} x^{i}+\frac{1}{2} \ln F .
$$

In this scheme, the exact form of the equation for the function $F$ is simply

$$
-\frac{1}{2} \partial^{2} F^{-1}+b^{i} \partial_{i} F^{-1}=0
$$

i.e. the $F$-model

$$
L_{F}=F(x) \partial u \bar{\partial} v+\partial x^{i} \bar{\partial} x_{i}+\alpha^{\prime} \mathcal{R}\left(\phi_{0}+b_{i} x^{i}+\frac{1}{2} \ln F\right)
$$

with $F$ satisfying (2.30) is Weyl invariant to all orders $\frac{12}{12}$ In this scheme the leading-order duality is exact since the leading-order dual to 2.31) is the $K$-model

$$
L_{K}=\partial u \bar{\partial} v+F^{-1}(x) \partial x^{i} \bar{\partial} x_{i}+\alpha^{\prime} \mathcal{R}\left(\phi_{0}+b_{i} x^{i}\right)
$$

11 Similar redefinitions of the metric appeared in the context of gauged WZW $\sigma$-models, relating a 'standard' scheme to a scheme where the leading-order solution is exact [10,11] (see also Section $6)$.

12 The $F$-model (2.31) considered on a flat $2 d$ background is thus UV finite on shell. It may be possible to prove this fact in a more direct way (without the need for an extra redefinition of the metric) using the manifestly covariant normal coordinate expansion in a way similar to how it was done for the WZW model in [28]. One would then still have to show that there exists a dilaton such that the condition of Weyl invariance (which is stronger than scale invariance [29]) is satisfied as well. 
which represents an exact string solution if $F$ solves (2.30) (cf. (2.20) with $\phi=0)$. In particular, we conclude that there exists a scheme in which the FS solution

$$
\begin{gathered}
F^{-1}=1+\frac{M}{r^{D-4}}, \quad D>4 \\
F^{-1}=1-M \ln r, \quad D=4, \quad r^{2} \equiv x_{i} x^{i},
\end{gathered}
$$

is a classical string solution to all orders in $\alpha^{\prime}$.

The conclusion about the existence of a scheme where the $F$-model (2.31) represents an exact string solution is consistent with the result of [13] that the particular $F$-model

$$
F^{-1}=\sum_{i=1}^{N} \epsilon_{i} \mathrm{e}^{\alpha_{i} \cdot x}, \quad \phi=\phi_{0}+\rho \cdot x+\frac{1}{2} \ln F
$$

(where the constants $\epsilon_{i}$ take values 0 or $\pm 1, \quad \alpha_{i}$ are simple roots of the algebra of a maximally non-compact Lie group $G$ of rank $N=D-2$ and $\rho=\frac{1}{2} \sum_{s=1}^{m} \alpha_{s}$ is half of the sum of all positive roots) can be obtained from a $G / H$ gauged WZW model. $H$ is a nilpotent subgroup of $G$ generated by $N-1$ simple roots (this condition on $H$ is needed to get models with one time direction). For example, the $D=4$ models are obtained for each of the rank 2 maximally non-compact groups $(S L(3), S O(2,2)$, etc.); for

$\epsilon_{i}=1, F^{-1}=\mathrm{e}^{\alpha_{1} \cdot x}+\mathrm{e}^{\alpha_{2} \cdot x}$ with the classical string propagation being determined by the Toda equation. As argued in [13], this background does not receive $\alpha^{\prime}$ corrections in the 'CFT' scheme: since the gauged subgroup is nilpotent, the action or $L_{0}$ operator of the coset model is not modified by $1 / k$-corrections except for the standard overall rescaling $k^{-1} \rightarrow\left(k+\frac{1}{2} c_{G}\right)^{-1}$ (see also Section 6$)$.

\subsection{Remarks}

We have discussed the $F$-model from the point of view of a perturbative path integral approach. One may try to give an alternative proof of its conformal invariance using the existence of the two chiral currents $J_{u}=F \partial u, \quad \bar{J}_{v}=F \bar{\partial} v$ to construct directly the conformal stress-energy tensor. At first sight, it appears this idea should not work since in contrast to the case of the WZW model here we do not have enough chiral currents. The $x^{i}$-currents do not look chiral since the $x^{i}$-equation of motion is not free and has an interaction potential proportional to $\partial_{i} F^{-1}$ (for example, this is the Toda equation in the case of the models in (2.34)

$$
\partial \bar{\partial} x_{i}=-\frac{1}{2} \partial_{i} F^{-1}(x) J_{u}(z) \bar{J}_{v}(\bar{z}) .
$$


However, extra chiral currents may still exist. This is illustrated by the example of the particular $D=3 F$-model with $F=e^{-2 b x}$ which is equivalent (see Section 5 ) to the $S L(2, R)$ WZW model and which thus must have extra chiral currents in addition to $J_{u}$ and $\bar{J}_{v}$. In fact, if we define

$$
J_{x}=\partial x+F v \partial u=\partial x+v J_{u}, \quad \bar{J}_{x}=\bar{\partial} x+F u \bar{\partial} v=\bar{\partial} x+u \bar{J}_{v},
$$

then the classical equations imply

$$
\partial \bar{J}_{v}=0, \quad \bar{\partial} J_{u}=0, \quad \partial \bar{J}_{x}=0, \bar{\partial} J_{x}=0,
$$

where we have used the fact that $F$ is a pure exponential. Extra chiral currents must also exist for the generic $D=3 F$-model since, as we shall show in Section 5 , it is equivalent to a gauged $S L(2, R) \times R / R$ WZW model, as well as for the the models (2.34) which can also be obtained from particular gauged WZW models [13].13

Another comment we would like to make is about possible supersymmetric generalisations. The model (2.1) has an obvious $n=1$ supersymmetric version with the fields $u, v, x^{i}$ replaced by $n=1$ superfields $\left(\mathcal{D} \equiv \frac{\partial}{\partial \theta}-\theta \frac{\partial}{\partial z}\right)$

$$
\int d^{2} z d^{2} \theta\left[F(\hat{x}) \mathcal{D} \hat{u} \overline{\mathcal{D}} \hat{v}+\left(G_{i j}+B_{i j}\right)(\hat{x}) \mathcal{D} \hat{x}^{j} \overline{\mathcal{D}} \hat{x}^{i}+\alpha^{\prime} \hat{\mathcal{R}} \phi(\hat{x})\right] .
$$

It would be interesting to formulate conditions on the functions of the $F$-model under which (2.1) admits $n>1$ generalisations. Such extended supersymmetric versions exist for the special $F$-models which correspond to gauged WZW models.

As for generic $F$-models, we can try to draw an analogy with the case of the $n=1$ supersymmetric gauged WZW theories. In supersymmetric WZW models there is no nontrivial shift of $k$ coming from the measure since the Jacobian ( $\hat{y}$ and $\hat{y}^{\prime}$ are superfields of opposite statistics)

$$
\int[d \hat{y}]\left[d \hat{y}^{\prime}\right] \exp \left\{-\int d^{2} z d^{2} \theta \hat{y}(\mathcal{D}+[A,]) \hat{y}^{\prime}\right\}
$$

is trivial [9]: the fermionic and bosonic $A$-dependent contributions cancel out. This is the reason why the corresponding $\sigma$-model couplings receive no $\alpha^{\prime}$-corrections [30,8,9]. A

13 The chiral currents in $\sigma$-models obtained by integrating out the 2 d gauge field in a gauged WZW model should be non-local when expressed directly in terms of the $\sigma$-model fields. 
similar conclusion is true for the $n=1$ super-generalisation of the determinant factor $\Omega$ in (2.10)

$$
\Omega[\hat{x}, \gamma]=\int[d \hat{u} d \hat{v}] \exp \left[-\int d^{2} z d^{2} \theta F(\hat{x}) \mathcal{D} \hat{u} \overline{\mathcal{D}} \hat{v}\right]
$$

Since $F(\hat{x}) \mathcal{D} \hat{u} \overline{\mathcal{D}} \hat{v}=(\mathcal{D}+f) \hat{u}^{\prime}(\overline{\mathcal{D}}+\bar{f}) \hat{v}^{\prime}, \quad f=-\frac{1}{2} \mathcal{D} \ln F, \bar{f}=-\frac{1}{2} \overline{\mathcal{D}} \ln F$, it is natural to expect that this determinant factor contains only the dilaton contribution and not the derivative $f \bar{f}$-term when defined in a supersymmetric way. Then the metric in (2.15) does not have the $\alpha^{\prime}$ - correction. This is certainly true for the particular $F$-models $(D=3$ and models in (2.34)) which are related to gauged WZW theories.

\section{New exact solutions in five and four dimensions}

We showed in the previous section that one could construct new exact solutions which were a hybrid of the gauged WZW models and the plane waves by using a gauged WZW model to describe the transverse space and adding $d u d v+K d u^{2}$ to the metric. The function $K$ must solve the scalar 'tachyonic' equation (with zero mass) in the transverse space. For a gauged WZW model, there exists a 'CFT scheme' where the tachyonic equation is simple (given by the zero mode part of the CFT Hamiltonian or $L_{0}$ ) while $G, B$ and $\phi$ may receive $\alpha^{\prime}$-corrections. By solving this simple equation, and using the known exact form of $G, B$ and $\phi$, one obtains new exact solutions.

\subsection{Five dimensions}

The simplest example of this construction starts with the $S U(2)$ WZW model. In this case the dilaton is constant, the metric is the standard round metric on $S^{3}$

$$
d s^{2}=d \xi^{2}+\sin ^{2} \xi d \Omega_{2}
$$

and $H_{i j k}=\epsilon_{i j k}$ is the volume form. In the CFT scheme, the metric and antisymmetric tensor have only a constant overall rescaling and the relevant equation for $K$ is just $\Delta K=$ 0 , where $\Delta$ is the Laplacian on $S^{3}$. Assuming $S O(3)$ symmetry, the general solution is $K=a+m \cot \xi$. The constants $a$ and $m$ can be absorbed into a redefinition of $u$ and $v$ so one obtains the following new exact solution:

$$
d s^{2}=d u d v+\cot \xi d u^{2}+d \xi^{2}+\sin ^{2} \xi d \Omega_{2}
$$


with $H_{i j k}=\epsilon_{i j k}$ and $\phi=$ const as before. This solution has singularities at the poles of the $S^{3}$. To interpret these singularities, it is useful to consider the dual $F$-model.

Starting with the general solution for $K$ and dualizing with respect to $u$ yields

$$
d s^{2}=(a+m \cot \xi)^{-1} d u d v+d \xi^{2}+\sin ^{2} \xi d \Omega_{2}
$$

Keeping the constants $a$ and $m$ is necessary to obtain the general solution, since they correspond to the freedom to dualize with respect to the symmetry which is a linear combination of translations of $u$ and $v$. In the $F$-model it is not possible to remove both $a$ and $m$ by redefining $u$ and $v$, but clearly $a$ can be set to be either \pm 1 or 0 by rescaling one of the coordinates. The dual of (3.2) is the special case of (3.3)

$$
d s^{2}=\tan \xi d u d v+d \xi^{2}+\sin ^{2} \xi d \Omega_{2}
$$

Since $u$ in (3.2) is timelike on one hemisphere and spacelike on the other and so is null at the equator, the dual with respect to $u$ (3.4) has an additional singularity there. It still has singularities at the poles, but in a neighborhood of these singularities, the solution approaches the five dimensional FS solution (see (1.3))

$$
d s^{2}=\frac{r}{r+M} d u d v+d r^{2}+r^{2} d \Omega_{2}
$$

Thus even though the transverse space is curved, the singularities introduced by adding an $S O(3)$ symmetric $K$ in (3.2) are just like the one in the background dual to the FS. This is not surprising since locally the transverse space is, of course, flat.

\subsection{Four dimensions}

To obtain a four dimensional solution one must start with a two dimensional conformal $\sigma$-model. Essentially the only non-trivial possibility is the $S L(2, R) / U(1)$ gauged WZW model which describes the two dimensional euclidean black hole. As discussed above, to construct this new solution we must use all the zero-mode information provided by the $S L(2, R) / U(1)$ coset: the exact metric and dilaton and the form of the tachyon equation. This will give us the all-order form of all the functions in the $D=4 \sigma$-model (2.2). The solution constructed in this way will be the generic $D=4 \mathrm{~K}$-model.

Let us first review what is known about the exact background fields of $S L(2, R) / U(1)$ model in the CFT scheme [7]. The metric and dilaton are given by

$$
d s^{2}=G_{i j} d x^{i} d x^{j}=d x^{2}+\frac{\tanh ^{2} b x}{1-p \tanh ^{2} b x} d \theta^{2}
$$




$$
\phi=\phi_{0}-\ln \cosh b x-\frac{1}{4} \ln \left(1-p \tanh ^{2} b x\right),
$$

where the parameters $b$ and $p$ are related by

$$
\begin{gathered}
p \equiv \frac{2}{k}, \quad \alpha^{\prime} b^{2}=\frac{1}{k-2}, \\
D-26+6 \alpha^{\prime} b^{2}=\frac{3 k}{k-2}-1-26=0, \quad D=2 .
\end{gathered}
$$

Since $\theta$ must be periodic, it is convenient to introduce the shifted dilaton

$$
\varphi \equiv 2 \phi-\frac{1}{2} \ln \operatorname{det} G
$$

The physical coupling in this case is $\exp \left(\frac{1}{2} \varphi\right)$ which is invariant under the leading order duality transformation. For the above solution, the shifted dilaton is simply

$$
\varphi=\varphi_{0}-\ln \sinh 2 b x
$$

Since in the CFT scheme the tachyonic equation has the standard uncorrected form, the function $K(x)$ must satisfy (see eq.(2.18); here $\phi$ is $u$-independent)

$$
-\frac{1}{2} \nabla^{2} K+\partial^{i} \phi \partial_{i} K=-\frac{1}{2 \sqrt{G} \mathrm{e}^{-2 \phi}} \partial_{i}\left(\sqrt{G} \mathrm{e}^{-2 \phi} G^{i j} \partial_{j}\right) K=0 .
$$

Observing that the measure factor is $\sqrt{G} \exp (-2 \phi)=c_{0} \sinh 2 r$ and assuming that $K$ depends only on $x$ and not on $\theta$ we find that the solution of (3.11) is simply

$$
K=a+m \ln \tanh b x .
$$

The constants $a, m$ can again be absorbed into a redefinition of $u$ and $v$, so that the full exact $D=4$ metric is 14

$$
d s^{2}=d u d v+\ln \tanh b x d u^{2}+d x^{2}+\frac{\tanh ^{2} b x}{1-p \tanh ^{2} b x} d \theta^{2},
$$

while the dilaton is unchanged. This metric is asymptotically flat, being a product of $D=2$ Minkowski space with a cylinder at infinity. Note that now the condition on $b$ is different than in the $D=2$ solution since there are two extra dimensions

$$
4-26+6 \alpha^{\prime} b^{2}=\frac{3 k}{k-2}-25=0 .
$$

14 We can generalise this solution by introducing a $u$-dependent dilaton. Then $K$ will get an extra piece $\sim x^{2}$ and will grow at large $x$ as in the plane wave case. 
Remarkably, the solution for $K$ (3.12) is the same in the 'leading-order' scheme where the metric and dilaton do not receive $\alpha^{\prime}$ corrections. The point is that the tachyon operator remains the same differential operator, it is only its expression in terms of the new $G, \phi$ that changes. Thus, in the 'leading-order' scheme we get the following exact $D=4$ solution

$$
\begin{gathered}
d s^{2}=d u d v+\ln \tanh b x d u^{2}+d x^{2}+\tanh ^{2} b x d \theta^{2}, \\
\phi=\phi_{0}-\ln \cosh b x, \quad \varphi=\varphi_{0}-\ln \sinh 2 b x .
\end{gathered}
$$

In addition to the covariantly constant null vector $\partial / \partial v$, this solution has two isometries corresponding to shifts of $u$ and $\theta$. Hence we can consider two different types of duals. Dualizing with respect to $\theta$ yields

$$
\begin{gathered}
d s^{2}=d u d v+\ln \tanh b x d u^{2}+d x^{2}+\operatorname{coth}^{2} b x d \theta^{2}, \\
\phi=\phi_{0}-\ln \sinh b x, \quad \varphi=\varphi_{0}-\ln \sinh 2 b x .
\end{gathered}
$$

For the two dimensional euclidean black hole, this duality can be viewed as a result of a coordinate shift $b x \rightarrow b x+i \pi / 2$, under which the solution remains real. This is no longer the case for the four dimensional solution (3.15) since $G_{u u}$ is unchanged under this duality.

To obtain the dual with respect to $u$ we again need to start with the general solution for $K$ (3.12). We get

$$
\begin{gathered}
d s^{2}=F(x) d u d v+d x^{2}+\tanh ^{2} b x d \theta^{2}, \quad B_{u v}=\frac{1}{2} F(x) \\
\phi=\phi_{0}-\ln \cosh b x+\frac{1}{2} \ln F, \quad F^{-1}=K=a+m \ln \tanh b x .
\end{gathered}
$$

In contrast to the euclidean $D=2$ black hole, the above $D=4 \mathrm{~K}$-model and $F$-model metrics in (3.15) and (3.17) have curvature singularities at $x=0$. The $F$-model (3.17) may have an additional curvature singularity at nonzero $x$ depending on the parameters $a$ and $m$. Let us choose $a=1, m=-M(M>0)$ so that these additional singularities are absent. We thus find

$$
\begin{gathered}
d s^{2}=(1-M \ln \tanh b x)^{-1} d u d v+d x^{2}+\tanh ^{2} b x d \theta^{2}, \\
B_{u v}=\frac{1}{2}(1-M \ln \tanh b x)^{-1}, \quad \phi=\phi_{0}-\ln \cosh b x-\frac{1}{2} \ln (1-M \ln \tanh b x) .
\end{gathered}
$$

The singularity at the origin of this solution is exactly of the same type that appears in the FS solution (see (1.3)): in $D=4$ we have $F^{-1}=1-M \ln r \rightarrow-M \ln r$ near $r=0$, while 
here $F^{-1}=1-M \ln \tanh b x \rightarrow-M \ln b x$ near $x=0$. As in the five dimensional example, the $F$-model (3.18) is completely equivalent to the fundamental string near $x=0$ 迆

$$
x \rightarrow 0: \quad d s^{2} \rightarrow(-M \ln b x)^{-1} d u d v+d x^{2}+(b x)^{2} d \theta^{2} .
$$

This behavior near $x=0$ would be the same if we had started with the metric (3.13) in the CFT scheme.

Moreover, the above $F$-model (3.18) can be viewed as an improved version of the FS solution in four dimensions. In $D=4$, the FS is given by (1.1) with $F^{-1}=1-M \ln r$. In addition to the usual singularity at $r=0$ there is another singularity outside the string at nonzero $r$. The solution we have just constructed (3.18) has the same singularity at the origin (and hence can be viewed as the field outside a fundamental string) but is regular elsewhere and even asymptotically flat. The original FS can be recovered by taking the limit $b \rightarrow 0$ which is consistent since the central charge condition is now imposed only at the level of the full $D=4$ solution (and can be satisfied, e.g., by adding 22 extra free degrees of freedom).

\section{Field redefinition ambiguity and structure of the effective action in $D=2$ and $D=3$}

The aim of this section is to discuss the general structure of the tree-level string theory effective action (EA) emphasizing a possibility to use the field redefinition freedom to put higher order $\alpha^{\prime n}$-corrections in the simplest form. In particular, we shall show that the EA can be chosen in such a form (a 'scheme') that all $\alpha^{\prime}$-corrections vanish once we specialise to the case of a $D=2$ background. In such a scheme the $D=2$ metric-dilaton EA is thus known explicitly, i.e. is given by the leading-order terms. There also exists a scheme in which the $D=3$ limit of the EA has all $\alpha^{\prime}$-corrections depending only on the derivatives of the dilaton but not on the curvature or antisymmetric tensor.

15 The reason why the two solutions agree is that near $x=0$ the dilaton is constant and thus the equation for $F:-\frac{1}{2} \nabla^{2} F^{-1}+\partial^{i} \phi \partial_{i} F^{-1}=0$ takes its FS form $\nabla^{2} F^{-1}=0$. 


\section{1. 'Scheme dependence' of the effective action}

Let us first recall a few basic facts about the string effective action [31, 32]. Given a tree-level string S-matrix (in $D=26$ ) we can try to reproduce its massless sector by a local covariant field-theory action $S(G, B, \phi)$ for the metric, antisymmetric tensor and dilaton. Subtracting the massless exchanges from the string scattering amplitudes and expanding the massive ones in powers of $\alpha^{\prime}$ gives an infinite series of terms in $S$ of all orders in $\alpha^{\prime}$.

The form of such action is not unique: a class of actions related by field redefinitions which are local, covariant, background-independent, power series in $\alpha^{\prime}$ (depending on dilaton only through its derivatives not to mix different orders of string loop perturbation theory) will correspond to the same string $S$-matrix. Given some representative in a class of equivalent EA's we refer to other equivalent actions as corresponding to different 'schemes'. The reason for this terminology is that the extremality conditions for the effective action are equivalent to the conditions of conformal invariance of the $\sigma$-model representing string action in a background [33, 34] and the related ambiguity in the $\sigma$-model Weyl anomaly coefficients or ' $\beta$-functions' can be interpreted as being a consequence of different choices of a renormalisation scheme [35]. This implies that some coefficients of the $\alpha^{\prime n}$-terms in the EA will be unambiguous (being fixed by the string $S$-matrix) while many others will be 'scheme-dependent'.

Though one possible way of determining the EA is to start with perturbative massless string scattering amplitudes on a flat $D=26$ background, $S$ must actually be backgroundindependent.16 In particular, its unambiguous coefficients are universal (e.g. they do not dependent on the dimension $D$ ). This is implied by the equivalence between the effective equations of motion and the string $\sigma$-model Weyl invariance conditions (which are background-independent). To make this equivalence precise in any dimension $D$ we need only to add to the EA one $D$-dependent ('central charge') term $\sim \int d^{D} x \sqrt{G} \exp (-2 \phi)(D-$ 26).17

16 After all, we expect the EA to be a result solving for the 'massive modes' in a hypothetical background-independent string field theory action.

17 There is actually a subtlety related to this term: it is not clear that one can have a consistent $\alpha^{\prime}$-perturbation theory if one is expanding near a vacuum with $D \neq 26$. For example, the linear dilaton background will involve a parameter of order $1 / \alpha^{\prime}$. This problem can be formally avoided by assuming that the central charge condition is imposed only at the very end and/or extra degrees of freedom are added to make total $D$ equal (or very close) to 26 . 
Given such a background-independent EA

$$
S=\int d^{D} x \sqrt{G} \mathrm{e}^{-2 \phi}\left\{\frac{2(D-26)}{3 \alpha^{\prime}}-\left[R+4\left(\partial_{\mu} \phi\right)^{2}-\frac{1}{12}\left(H_{\mu \nu \lambda}\right)^{2}\right]+O\left(\alpha^{\prime}\right)\right\}
$$

it would be useful to choose a scheme (i.e. the values of ambiguous coefficients) in which $S$ has the simplest possible form. 18 For example, the correspondence with the Weyl anomaly coefficients of a string $\sigma$-model implies that there exists a scheme in which (4.1) does not contain other higher-order dilatonic terms. This follows also from the general argument [36] based on the path integral representation for the EA [32] and was checked directly at the $\alpha^{\prime}$-order [37.38] by comparing with string $S$-matrix. We now show that in three dimensions one can do just the opposite, i.e., have only dilaton terms as higher order corrections.

\subsection{Effective action in $D \leq 3$}

It is possible to arrive at a more definitive conclusion about a simplest possible scheme by specialising to the low dimensional cases of $D=2$ and $D=3$. More precisely, we would like to find an EA (defined for general $D$ ) such that its $\alpha^{\prime n}$-terms take a simple form in the limit $D \rightarrow 2,3$.

Given that (4.1) is background-independent (in particular, its higher-order coefficients do not depend on $D)$ we are free to take $(G, B)$ in (4.1) to correspond to a generic $D=2$ or $D=3$ background. Since the basic fields $(G, B)$ are second rank tensors, higher order terms which involve 'irreducible' contractions of tensors of rank greater than two cannot be altered by field redefinitions. But in $D \leq 3$ the Riemann tensor can be expressed in terms of the Ricci tensor, and $H_{\mu \nu \lambda}=\epsilon_{\mu \nu \lambda} H$. Thus all possible covariant structures in the EA will have the 'reducible' form of products of scalars, vectors, or at most, second-rank tensors.

This is a necessary condition for a higher order term to be removed by a field redefinition, but it is not sufficient. It has been shown [39] that some combinations of a priori ambiguous coefficients in the EA are actually redefinition-invariant (unambiguous)

18 As usual in field theory, one is trying to fix the freedom of local field redefinitions in such a way that to have the simplest possible action reproducing given $S$-matrix. For example, one would prefer to reproduce the graviton scattering amplitudes of the Einstein theory by the Einstein action but not by a complicated action $\int d^{4} x \sqrt{G^{\prime}} R\left(G^{\prime}\right), G_{\mu \nu}^{\prime}=G_{\mu \nu}+\alpha^{\prime} R_{\mu \nu}$, which contains all powers of the curvature. 
and thus are uniquely determined by the string $S$-matrix. In fact, it is easy to show that one cannot find a scheme in which there is no $\alpha^{\prime}$-term in the $D=3$ EA. Suppose $H=0$ for simplicity. Then in the standard scheme, the $\alpha^{\prime}$ correction to the bosonic string EA is simply

$$
S_{1}(G, B, \phi)=a_{0} \int d^{D} x \sqrt{G} \mathrm{e}^{-2 \phi}\left(R_{\mu \nu \kappa \lambda}\right)^{2}
$$

In three dimensions, $\left(R_{\mu \nu \kappa \lambda}\right)^{2}=4\left(R_{\mu \nu}\right)^{2}-R^{2}$. Under a field redefinition, the action changes by a term proportional to the leading order equations of motion. So if $S_{1}$ can be removed by a field redefinition, it must vanish when the equations of motion are satisfied (up to surface terms). Consider first the Ricci term $\int d^{D} x \sqrt{G} \mathrm{e}^{-2 \phi}\left(R_{\mu \nu}\right)^{2}$. Using the low energy equations of motion with $H=0$ and integrating by parts, one can show that this is a total divergence in any dimension! But the scalar curvature contribution $\int d^{3} x \sqrt{G} \mathrm{e}^{-2 \phi} R^{2}$ turns out to be nonzero in general. Thus in $D=3$ the order $\alpha^{\prime}$ term in the EA cannot be removed completely, although its form can be altered. However in $D=2$, one can write $\left(R_{\mu \nu \kappa \lambda}\right)^{2}=2\left(R_{\mu \nu}\right)^{2}$ so this term now vanishes when the leading order equations are satisfied. Using the results of [37] one can show that this term can indeed be removed by a field redefinition.

So far we have discussed just the first order $\alpha^{\prime}$ correction. What can one say more generally? Consider first the $D=2$ case where $H_{\mu \nu \lambda}$ automatically vanishes. Suppose we compute the scattering amplitudes for the dilaton and graviton (in general $D$ ) directly in the string frame where the dilaton and graviton mix in the propagator. Since there are no transverse degrees of freedom for the string in $D=2$, there are no dynamical degrees of freedom in the $(G, \phi)$ system, and the limit $D \rightarrow 2$ of the scattering amplitudes is trivial. That means that the on-shell limits of unambiguous terms in the EA must vanish identically. Hence there exists a choice of the EA (in generic $D$ ) such that higher order terms in it vanish in the $D \rightarrow 2$ limit.

A similar statement is not true in $D=3$ since there is one transverse degree of freedom for the string which could yield higher order corrections to the scattering amplitudes and hence to the EA. However one can express these corrections solely in terms of the dilaton. To see this, consider the exact equations of motion for $G_{\mu \nu}, B_{\mu \nu}$, and $\phi$ in some scheme:

$$
\begin{gathered}
R_{\mu \nu}+\frac{1}{2} H^{2} G_{\mu \nu}+2 \nabla_{\mu} \nabla_{\nu} \phi=\sum_{n=1}^{\infty} \alpha^{\prime n} T_{\mu \nu}^{n}, \\
\nabla_{\mu}\left(e^{-2 \phi} H\right)=\sum_{n=1}^{\infty} \alpha^{\prime n} V_{\mu}^{n}
\end{gathered}
$$




$$
4 \nabla^{2} \phi-4(\nabla \phi)^{2}+R+\frac{1}{2} H^{2}-\frac{2(D-26)}{3 \alpha^{\prime}}=\sum_{n=1}^{\infty} \alpha^{\prime n} S^{n},
$$

where $T_{\mu \nu}^{n}, V_{\mu}^{n}$, and $S^{n}$ are the higher order correction terms and we have used the fact that $H_{\mu \nu \lambda}=\epsilon_{\mu \nu \lambda} H$ in $D=3$ (and assumed Minkowski signature). Since we are interested in solving these equations perturbatively in $\alpha^{\prime}$, we can proceed as follows. Start with $n=1$ and use (4.5) to replace the $H^{2}$ terms in $T_{\mu \nu}^{1}, V_{\mu}^{1}$, and $S^{1}$ (and the left hand side of (4.3)) by dilaton and curvature terms. Then use (4.4) to replace $(\nabla H)^{2}$ terms by the dilaton and curvature. Finally, use (4.3) to replace all the curvature terms by derivatives of the dilaton. This will, of course, change the form of the correction terms for $n>1$ but it will ensure that the $n=1$ terms only involve the dilaton. One can now repeat this procedure for each $n$. In this way, one can express all the correction terms solely in terms of derivatives of the dilaton. The action which reproduces this form of the exact equations will then have only dilaton terms as higher order corrections.

\subsection{Discussion}

Let us discuss some implications of the above remarks. Since in $D=2$ there is a scheme in which all $\alpha^{\prime}$ corrections to the EA vanish, all backgrounds which solve the leading-order equations are in fact exact solutions. This conclusion is not so surprising: the $D=2$ 'black hole' background [22,5] represents the generic solution of the leadingorder equations, and given that the corresponding CFT is known $(S L(2, R) / U(1)$ coset [5]) one can find explicitly [36] a local covariant background-independent redefinition from the 'CFT scheme' [7] (where the background fields are $\alpha^{\prime}$ - dependent) to the 'leading-order' scheme. It also follows that in this scheme the $D=2 \sigma$-model Weyl anomaly coefficients just have their leading-order form.19

As for $D=3$, in the scheme where $\alpha^{\prime}$-corrections are proportional to the derivatives of $\phi$, the solutions of the leading-order equations which have constant $\phi$ remain exact to all orders. It is easy to see that the only leading-order solution with constant $\phi$ in $D=3$ is the constant curvature anti-de Sitter space with the parallelising $H_{\mu \nu \lambda}$-torsion corresponding

19 One may be tempted to draw a conclusion that there exists a scheme where the $\beta$-functions of a generic $D=2 \sigma$-model also have just the leading-order form. This may not necessarily be the case since the field redefinitions implied in our argument are more general (involving the dilaton) than the redefinitions corresponding to the freedom of choice of a renormalisation scheme in the standard $\sigma$-model $\beta$-functions. It may be of interest to understand this question further, e.g., in connection with the RG flow in some $2 \mathrm{~d} \sigma$-models 40 ]. 
to the $S L(2, R)$ WZW model or its possible cosets over discrete subroups (in particular, the $D=3$ black hole of [21]). For solutions with nonconstant $\phi$, the best that we can hope for is to find a scheme in which a particular leading-order solution does not receive $\alpha^{\prime}$ corrections. This was shown [11] to be the case (to $\alpha^{\prime 2}$ order) for the charged black string background [18], i.e. $S L(2, R) \times R / R$ coset model. In the next section we will find that there exists a scheme where the leading order solution for the $D=3 F$-model (2.1), 2.30) is also conformal to the next order in $\alpha^{\prime}$. This will provide a perturbative check of the general path integral argument of Section 2.4.

Another implication concerns an exact form of the abelian duality transformations: leading-order duality [19] is the symmetry of the leading-order terms in the EA [41,20] and thus is the exact symmetry in the simplest scheme in $D=2$. In fact, we have checked directly that while for a general $D$ there does not exist a scheme in which the leading order duality remains a symmetry at $\alpha^{\prime}$-order without been modified by the derivative $O\left(\alpha^{\prime}\right)$-term [20], such scheme does exist in $D=2.20$

As for $D \geq 4$, here the massless sector of string $S$-matrix is non-trivial so no simple scheme should be expected to exist.

\section{F-model in three dimensions}

The first non-trivial example of the $F$-model is in $D=3$. If $b=0$, the equation for $F(2.30)$ just says that $F^{-1}$ is a linear function of $x$. Since the transverse space here is one-dimensional, we can absorb the two free parameters and write the solution as

$$
F^{-1}=x, \quad \phi=\phi_{0}-\frac{1}{2} \ln x
$$

This is the formal $D=3$ analog of the FS solution (1.3). The corresponding dual $K$-model

$$
d s^{2}=d u d v+x d u^{2}+d x^{2}, \quad \phi=\phi_{0},
$$

describes a flat spacetime.

The general solution of $(2.30)$ with $b \neq 0$ is 21

$$
F^{-1}=a+m \mathrm{e}^{2 b x},
$$

20 In the case of the $D=2$ black hole solution this was observed in 36].

21 We shall assume that $b<0$ so that $x \rightarrow+\infty$ corresponds to the asymptotically flat region. 


$$
\phi=\phi_{0}+b x-\frac{1}{2} \ln \left(a+m \mathrm{e}^{2 b x}\right) .
$$

(The solution (5.1) is recovered in the limit $b \rightarrow 0$ provided one takes $a$ and $m$ to infinity such that $a+m$ and $m b$ are kept fixed.) This solution is closely related to the $S L(2, R)$ WZW model. One way to see this is to note that in the limit $a \rightarrow 0$, the $F$-model (2.1) becomes equivalent to the $S L(2, R)$ WZW model written in the Gauss decomposition parametrisation

$$
\begin{gathered}
g=\left(\begin{array}{ll}
1 & u \\
0 & 1
\end{array}\right)\left(\begin{array}{cc}
\mathrm{e}^{r} & 0 \\
0 & \mathrm{e}^{-r}
\end{array}\right)\left(\begin{array}{cc}
1 & 0 \\
v & 1
\end{array}\right), \\
L_{w z w}=k\left(\partial r \bar{\partial} r+\mathrm{e}^{-2 r} \partial u \bar{\partial} v\right), \quad r=b x+\frac{1}{2} \ln m, \quad \alpha^{\prime} b^{2}=1 / k .
\end{gathered}
$$

As we have noted earlier, the actual value of $a$ is not physical since one can rescale $F$ by simply rescaling one of the coordinates $u$ or $v$. The values which yield geometrically different solutions are $a=0, \pm 1$.

Another connection between the $F$-model and the $S L(2, R)$ WZW model is through their duals. The $K$-model dual to (5.3) is

$$
L_{K}=\partial u \bar{\partial} v+\left(a+m \mathrm{e}^{2 b x}\right) \partial u \bar{\partial} u+\partial x \bar{\partial} x+\alpha^{\prime} \mathcal{R}\left(\phi_{0}+b x\right) .
$$

By the coordinate transformation $v \rightarrow v-a u$ and a rescaling of $u$ and $v$ this becomes

$$
L_{K}=\partial u \bar{\partial} v+\mathrm{e}^{2 b x} \partial u \bar{\partial} u+\partial x \bar{\partial} x+\alpha^{\prime} \mathcal{R}\left(\phi_{0}+b x\right)
$$

which is obviously $u$-dual to the $S L(2, R)$ WZW model (5.6). In other words, the $F$-model (5.3) is related to the $S L(2, R)$ WZW model by dualizing with respect to one symmetry and dualizing back with respect to another symmetry. This implies that the $D=3 F$ model (5.3) is an $O(2,2)$ rotation of the $S L(2, R)$ WZW model (5.6).22 This does not prove that the $F$-model is equivalent to the $S L(2, R)$ WZW model since dual models are equivalent only if the symmetry direction is compact; but if $u$ is periodically identified, the coordinate transformation $v \rightarrow v-a u$ is not globally valid.

We shall now show that the $F$-model with $b \neq 0$ (5.3) can, in fact, be derived from an $S L(2, R) \times R / R$ gauged WZW model. Let us first note that the standard lorentzian $D=2$ black hole [5] can be obtained by gauging the following global symmetry of the

22 In any dimension $D$, the $F$-model with function $F$ is related to the $F$-model with $F^{\prime}=$ $\left(F^{-1}+a\right)^{-1}, \quad a=$ const by the $\mathrm{O}(2,2)$ rotation (with the dilatons being the same). Obviously, if $F$ is a solution of (2.30) the same is true for $F^{\prime}$. 
$S L(2, R)$ WZW action in the Gauss decomposition parametrisation (5.6): $r^{\prime}=r+\epsilon, u^{\prime}=$ $e^{\epsilon} u, v^{\prime}=e^{\epsilon} v$. The gauged action is

$$
L_{g w z w}=k\left[(\partial r+A)(\bar{\partial} r+\bar{A})+\mathrm{e}^{-2 r}(\partial u+A u)(\bar{\partial} v+\bar{A} v)\right] .
$$

Fixing the gauge $r=0$ and solving for $A, \bar{A}$ first we finish with

$$
L_{b h}=k \frac{\partial u \bar{\partial} v}{1+u v}-\frac{1}{2} \mathcal{R} \ln (1+u v) .
$$

In contrast to the $F$-model here one cannot easily integrate over $u, v$. An equivalent expression is found by fixing the gauge as $u v=1, u=e^{t}$. The resulting metric is then given by $d s^{2}=k\left(1+e^{2 r}\right)^{-1}\left(-d t^{2}+d r^{2}\right)$.

Introducing an extra field $y$ and gauging independently the 'left' and 'right' subgroups of $S L(2, R) \times R$ (generated by the positive and negative roots as in [13], i.e., corresponding to the shifts of $u$ and $v$ in (5.5)) we get (cf. (5.9))

$$
L_{g w z w}=k\left[\partial r \bar{\partial} r+\mathrm{e}^{-2 r}(\partial u+\lambda A)(\bar{\partial} v+\nu \bar{A})\right]+k(\partial y+\rho A)(\bar{\partial} y+\rho \bar{A}) .
$$

Here the constants $\lambda, \nu, \rho$ correspond to a selection of a particular subgroup we are gauging (the action is invariant under: $u^{\prime}=u-\lambda \epsilon, v^{\prime}=v-\nu \epsilon, y^{\prime}=y-\rho \epsilon, A^{\prime}=A+\partial \epsilon, \bar{A}^{\prime}=$ $\bar{A}+\bar{\partial} \epsilon$ ). Fixing $y=0$ as a gauge and solving for $A, \bar{A}$ we finish with the $F$-model (see also Appendix C)

$$
F^{-1}=a+\mathrm{e}^{2 r}, \quad \phi=\phi_{0}+r+\frac{1}{2} \ln F, \quad a \equiv \frac{\lambda \nu}{\rho^{2}} .
$$

This model is equivalent to (5.3) under the same identification as in (5.6): $r=b x+$ $\frac{1}{2} \ln m, \alpha^{\prime} b^{2}=1 / k$. Given the freedom of rescaling $u, v$ and shifting $r$ the only non-trivial values of $a$ are again $0,+1,-1$. $a=0$ (i.e. the limit $\rho=\infty$ or $\lambda=0$ or $\nu=0$ ) gives back the $S L(2, R)$ WZW model.

Gauging the subgroup of $S L(2, R) \times R$ which is a straightforward extension of the black-hole one in (5.9) $\left(r^{\prime}=r+\epsilon, u^{\prime}=e^{\epsilon} u, v^{\prime}=e^{\epsilon} v, y^{\prime}=y-\rho \epsilon\right)$ and fixing the gauge $u=v^{-1}=e^{t}$ one can show that the corresponding $S L(2, R) \times R / R$ gauged WZW model yields the following charged black string background [18]

$$
d s^{2}=-\left(1-\frac{M}{r^{\prime}}\right) d t^{2}+\left(1-\frac{Q^{2}}{M r^{\prime}}\right) d y^{2}+\left(1-\frac{M}{r^{\prime}}\right)^{-1}\left(1-\frac{Q^{2}}{M r^{\prime}}\right)^{-1} \frac{k d r^{\prime 2}}{4 r^{\prime 2}},
$$




$$
B_{y t}=\frac{Q}{r^{\prime}}, \quad \phi=\phi_{0}-\frac{1}{2} \ln r^{\prime}, \quad \phi_{0}=-\frac{1}{4} \ln k
$$

where $M$ and $Q$ represent the charge and mass per unit length. This gauged WZW model only yields solutions with $Q<M$. However, given (5.13), one can clearly take the extremal limit $Q=M$ to obtain 23

$$
d s^{2}=\left(1-\frac{M}{r^{\prime}}\right)\left(-d t^{2}+d y^{2}\right)+\frac{k d r^{\prime 2}}{4\left(r^{\prime}-M\right)^{2}}
$$

Letting $b x$ denote the proper radial distance

$$
b x=-\frac{1}{2} \ln \left(r^{\prime}-M\right)
$$

one finds that the extremal black string is precisely the $F$-model (5.12) or (5.3) where the parameters are related by $a=1, m=M, \alpha^{\prime} b^{2}=1 / k$. As we have just seen, one can obtain this solution directly as a gauged WZW model by gauging a different subgroup of $S L(2, R) \times R$.

The relation between the $F$-model and black string clarifies the causal structure of the former. It was shown in [18 that the extremal three dimensional black string (5.14) has a horizon at $r^{\prime}=M$ but no singularity. The correct extension across the horizon is not to take $r^{\prime}<M$ but to use a new radial coordinate $\eta^{2}=r^{\prime}-M$. The $F$-model in the form (5.3) just covers the region outside the horizon and is incomplete.

In Section 2 we have found that all $F$-models are exact solutions in some scheme. How this is consistent with the fact that $D=3 F$-model is equivalent to a gauged WZW

23 The parametrisation of the charged black string background used in 42]

$$
d s^{2}=-\frac{z-q-1}{z} d t^{2}+\frac{z-q}{z} d x^{2}+\frac{d z^{2}}{4(z-q-1)(z-q)}
$$

is related to (5.13) by $z=\sqrt{k} r^{\prime}, \quad M=q / \sqrt{k}, Q^{2}=q(1+q) / k$, so that the extremal limit corresponds to $q \rightarrow \infty, k \rightarrow \infty, M=Q=q / \sqrt{k}=$ fixed. Let us mention also that in the Euler angle parametrisation of $S L(2, R) g=\mathrm{e}^{\frac{i}{2} \theta_{L} \sigma_{2}} \mathrm{e}^{\frac{1}{2} \tilde{r} \sigma_{1}} \mathrm{e}^{\frac{i}{2} \theta_{R} \sigma_{2}}, \theta_{L}=\theta+\tilde{\theta}, \theta_{R}=\tilde{\theta}-\theta$, the black string metric is 11]

$$
d s^{2}=\frac{1}{4} d \tilde{r}^{2}+(1+q) \frac{C-1}{C+1+2 q+2 b} d \theta^{2}-q \frac{C+1}{C+1+2 q} d \tilde{\theta}^{2}, \quad C=\cosh \tilde{r} .
$$

It is related to the above one in terms of $(z, x, t)$ by $2 z=C+1+2 q, i t=(1+q)^{1 / 2} \theta, \quad i x=-q^{1 / 2} \tilde{\theta}$, i.e. $\theta, \tilde{\theta}$ are to be infinitely rescaled and $\tilde{r}$ shifted in the extremal limit. 
model? First, there exists a scheme in which the leading order solution for the general charged black string (5.13) remains a solution to the next order in $\alpha^{\prime}$ [11]. In particular, this is true in the extremal limit. The above equivalence then implies that the general $F$-model in three dimensions is also exact to order $\alpha^{\prime}$ in the same scheme.24

Moreover, by taking the extremal limit of the exact expressions for the charged black string in the CFT scheme 42, 11] one finds that there are no genuine $\alpha^{\prime}$-corrections in this case (all dependence on $1 / k$ can be absorbed into rescalings of the coordinates). This is easy to see directly from (5.11). The origin of the $1 / k$ corrections to the $\sigma$-model backgrounds corresponding to gauged WZW models is in different renormalisation of the coefficients $\left(k \rightarrow k+\frac{1}{2} c_{G}\right.$ and $\left.k \rightarrow k+\frac{1}{2} c_{H}\right)$ in front of the group and subgroup parts of the action defined in the CFT scheme. The CFT scheme analog of (5.11) thus has the coefficient $k$ of the first $S L(2, R)$ two terms replaced by $k-2$ while the coefficient $k$ of the last $R$-subgroup term remains unrenormalised. That means that to find the exact background fields the constant $\rho^{2}$ and thus $a$ in (5.12) are to be replaced by $\rho^{\prime 2}=\rho^{2} \frac{k}{k-2}$ and $a^{\prime}=a\left(1-\frac{2}{k}\right)$. But as was already mentioned above, $a$ can be rescaled by a coordinate transformation. We conclude that like the $F$-model (2.34) obtained by the nilpotent gauging, the $D=3$ $F$-model does not receive non-trivial $\alpha^{\prime}$-corrections not only in the leading-order scheme but also in the CFT scheme.

\section{Relation between solutions in different schemes}

Let us now examine in more detail the relation between exact solutions in different schemes. Since $F$-models are in many respects similar to gauged WZW models 55 we shall start with some general comments on exact backgrounds corresponding to gauged WZW models.

24 We have also checked this directly starting with the $F$-model and repeating the computation in [11]. We would like to point out a misprint in eq. (4.33) of [11]: it should contain an extra term $-\nabla^{2} S$.

25 In particular, the integral over $u, v$ in the former is similar to the integral over $A, \bar{A}$ in the latter. 


\subsection{Solutions corresponding to gauged WZW models}

The classical gauged WZW action can be represented as

$$
I_{g w z w}=k I_{w z w}\left(h^{-1} g \tilde{h}\right)-k I_{w z w}\left(h^{-1} \tilde{h}\right), \quad A=h \partial h^{-1}, \quad \bar{A}=\tilde{h} \bar{\partial} \tilde{h}^{-1},
$$

i.e. as a difference of the two WZW actions for the total group $G$ and the gauged subgroup $H$. This representation implies that the gauged WZW model is a conformal theory. Fixing a gauge on $g$ and changing the variables to $g^{\prime}=h^{-1} g \tilde{h}, h^{\prime}=h^{-1} \tilde{h}$ we get a $\sigma$-model on the group space $G \times H$ which is conformal to all orders in a particular 'leading-order' scheme. That means that the 1-loop group space solution remains exact solution in that scheme. Replacing (6.1) with the 'quantum' action with renormalised levels $k \rightarrow k+\frac{1}{2} c_{G}$ and $k \rightarrow k+\frac{1}{2} c_{H}$ does not change this conclusion. This replacement corresponds to starting with the theory formulated in the 'CFT' scheme in which, e.g., the exact central charge of the WZW model is reproduced by the first non-trivial correction [10,11] and the metric $\left(k+\frac{1}{2} c_{G}\right) G_{\mu \nu}$ is the one that appears in the CFT Hamiltonian $L_{0}$ considered as a Klein-Gordon operator.

To obtain the corresponding $\sigma$-model in the 'reduced' $G / H$ configuration space (with coordinates being parameters of gauge-fixed $g$ ) one needs to integrate out $A, \bar{A}$ (or, more precisely, the WZW fields $h$ and $\tilde{h}$ ). This is a non-trivial step and the form of the result depends on a choice of a scheme in which the original 'extended' $(g, h, \tilde{h})$ WZW theory is formulated.

Suppose first the latter is taken in the leading-order scheme with the action (6.1). Then the result of integrating out $A, \bar{A}$ can be found by using a matrix generalisation of the formulas (2.10),(2.12),(2.14). If the $O\left(A^{2}\right)$ term in (6.1) is written as $\int d^{2} z F_{a b} A^{a} \bar{A}^{b}$, $F_{a b}=\operatorname{Tr}\left(g^{-1} T_{a} g T_{b}-\delta_{a b}\right)$, then under a specific assumption about the measure the correction to the action is 26

$$
\Delta I=-\frac{1}{2 \pi} \int d^{2} z \partial(\ln \operatorname{det} F) \bar{\partial}(\ln \operatorname{det} F)-\frac{1}{8 \pi} \int d^{2} z \sqrt{\gamma} R^{(2)} \ln \operatorname{det} F .
$$

26 In general, the derivative term in $\Delta I$ will have the form $\operatorname{Tr}\left[\partial f_{1}(F) \bar{\partial} f_{2}(F)\right]$ where $f_{i}$ are functions of the matrix $F$. The choice of the measure should be consistent with the assumption that the resulting $\sigma$-model should be formulated in a target space covariant way. In particular, the resulting dilaton should be the one that can be also obtained by solving directly the covariant $\sigma$-model conformal invariance equations. 
The resulting $\sigma$-model metric and dilaton are then given by (cf. (2.15))

$$
G_{\mu \nu}^{\prime}=G_{\mu \nu}-2 \alpha^{\prime} \partial_{\mu} \phi \partial_{\nu} \phi, \quad \phi=\phi_{0}-\frac{1}{2} \ln \operatorname{det} F
$$

where $G_{\mu \nu}$ is the metric obtained by solving for $A, \bar{A}$ at the classical level and $\phi_{0}$ is the original constant dilaton. Since the $\alpha^{\prime}$-term in the metric can be eliminated by a field redefinition we conclude that there exists a leading-order scheme in which the leading-order gauged WZW $\sigma$-model background $(G, B, \phi)$ remains an exact solution. The leading-order scheme for the ungauged WZW $\sigma$-model is thus related to the leading-order scheme for the gauged WZW $\sigma$-model by an extra $2 \alpha^{\prime} \partial_{\mu} \phi \partial_{\nu} \phi$ redefinition of the metric. This provides a general explanation for the observations in [10,11] about the existence of a leading-order scheme for particular $D=2,3$ gauged WZW models.

If instead we start with the $(g, h, \tilde{h})$ WZW theory in the CFT scheme, i.e with the action

$$
I_{g w z w}=\left(k+\frac{1}{2} c_{G}\right)\left[I_{w z w}\left(h^{-1} g \tilde{h}\right)-\frac{k+\frac{1}{2} c_{H}}{k+\frac{1}{2} c_{G}} I_{w z w}\left(h^{-1} \tilde{h}\right)\right],
$$

then the resulting $\sigma$-model couplings will explicitly depend on $1 / k$ (and will agree with the coset CFT operator approach results [7, 8, 9] ). While in the WZW model the transformation from the CFT to the leading order scheme is just a simple rescaling of couplings, this transformation becomes non-trivial at the level of gauged WZW $\sigma$-model. It is the 'reduction' of the configuration space resulting from integration over the gauge fields $A, \bar{A}$ that is responsible for a complicated form of the transformation law between the 'CFT' and 'leading-order' schemes in the gauged WZW $\sigma$-models (in particular, this transformation involves dilaton terms of all orders in $1 / k$, see Section 6.2 below).

An exception is provided by the $\sigma$-models (2.34) obtained by nilpotent gauging: here the second term in (6.4) is absent by construction [13]. The background fields do not receive non-trivial $1 / k$ corrections even in the $\mathrm{CFT}$ scheme, i.e. the relation between the leading-order and CFT schemes is equivalent to the one for the ungauged WZW model. The same is true for the $D=3 F$-model or the extremal limit of the $S L(2, R) \times R / R$ coset.

\subsection{Transformations between different schemes}

Let us now discuss how the above remarks are supported by the direct perturbative analysis. There exists a simple ('standard') scheme in which the order $\alpha$ ' effective action has the form [37]

$$
S=\int d^{D} x \sqrt{G} \mathrm{e}^{-2 \phi}\left\{\frac{2(D-26)}{3 \alpha^{\prime}}-\left[R+4 \nabla^{2} \phi-4(\nabla \phi)^{2}-\frac{1}{12}\left(H_{\mu \nu \lambda}\right)^{2}\right]\right.
$$




$$
\begin{gathered}
-\frac{1}{4} \alpha^{\prime}\left[R_{\mu \nu \lambda \kappa}^{2}-\frac{1}{2} R^{\mu \nu \kappa \lambda} H_{\mu \nu}^{\rho} H_{\kappa \lambda \rho}\right. \\
\left.\left.+\frac{1}{24} H_{\mu \nu \lambda} H_{\rho \alpha}^{\nu} H^{\rho \sigma \lambda} H_{\sigma}{ }^{\mu \alpha}-\frac{1}{8}\left(H_{\mu \alpha \beta} H_{\nu}{ }^{\alpha \beta}\right)^{2}\right]+O\left(\alpha^{\prime 3}\right)\right\} .
\end{gathered}
$$

The redefinition leading from this 'standard' scheme to the the leading-order scheme in which the parallelizable (group) space with $\phi=$ const is automatically a solution of the conformal invariance equations (to order $\alpha^{\prime}$ ) is [37]

$$
\begin{gathered}
G_{\mu \nu}^{(\text {lead })}=G_{\mu \nu}^{(\text {stand })}+\frac{1}{2} \alpha^{\prime} H_{\mu \nu}^{2}+O\left(\alpha^{\prime 2}\right), \\
B_{\mu \nu}^{(\text {lead })}=B_{\mu \nu}^{(\text {stand })}+O\left(\alpha^{\prime 2}\right), \quad \phi^{(\text {lead })}=\phi^{(\text {stand })}+O\left(\alpha^{\prime}\right),
\end{gathered}
$$

where $H_{\mu \nu}^{2}=H_{\mu \alpha \beta} H_{\nu}{ }^{\alpha \beta}$. As discussed above, this scheme should differ from the leadingorder scheme in which the gauged WZW background fields do not receive $\alpha^{\prime}$ corrections by the dilatonic term in (6.3). In fact, the 'leading-order' scheme in which the $[S L(2, R) \times R] / R$ gauged WZW background remains a solution at the $\alpha^{\prime}$-order is related to the 'standard' scheme by 11

$$
\begin{gathered}
G_{\mu \nu}^{(l e a d)}=G_{\mu \nu}^{(\text {stand })}+\frac{1}{2} \alpha^{\prime} H_{\mu \nu}^{2}-2 \alpha^{\prime} \partial_{\mu} \phi \partial_{\nu} \phi+O\left(\alpha^{\prime 2}\right) \\
B_{\mu \nu}^{(l e a d)}=B_{\mu \nu}^{(\text {stand })}+O\left(\alpha^{\prime 2}\right), \quad \phi^{(l e a d)}=\phi^{(\text {stand })}+\frac{1}{32} \alpha^{\prime}\left(H_{\mu \nu \lambda}\right)^{2}+\frac{1}{8} \alpha^{\prime} R+O\left(\alpha^{\prime 2}\right) .
\end{gathered}
$$

The two $\alpha^{\prime}$-terms in (6.7) thus have clear interpretation: the first $\left(H_{\mu \nu}^{2}\right)$ leads to the leading-order scheme for the ungauged WZW model while the second $\left(\partial_{\mu} \phi \partial_{\nu} \phi\right)$ is related to the derivative term (6.2) in the determinant factor which results from the integration over the gauge fields in the gauged WZW model.27

At the same time, the transformation between the standard scheme and the CFT scheme (in which the background fields receive corrections to all orders in $1 / k$ ) was found (for the $D=3[S L(2, R) \times R] / R$ background) to be [11]

$$
\begin{gathered}
G_{\mu \nu}^{(c f t)=G_{\mu \nu}^{(s t a n d)}}+\frac{1}{2} \alpha^{\prime} H_{\mu \nu}^{2}-2 \alpha^{\prime}(\partial \phi)^{2} G_{\mu \nu}+\alpha^{\prime} \nabla^{2} \phi G_{\mu \nu}+O\left(\alpha^{2}\right) \\
B_{\mu \nu}^{(c f t)}=B_{\mu \nu}^{(s t a n d)}+\alpha^{\prime} \nabla^{\lambda} \phi H_{\mu \nu \lambda}+O\left(\alpha^{\prime 2}\right)
\end{gathered}
$$

27 The transformation of the dilaton in (6.8) does not seem to have a simple interpretation since we do not explicitly know how the dilaton is defined in the 'standard' scheme compared to the leading-order scheme. 


$$
\phi^{(c f t)}=\phi^{(s t a n d)}+\frac{1}{12} \alpha^{\prime}\left(H_{\mu \nu \lambda}\right)^{2}+\frac{3}{8} \alpha^{\prime} R+O\left(\alpha^{\prime 2}\right) .
$$

The relation between the leading order and CFT schemes obtained in the case of $D=2$ $S L(2, R) / R$ model is 10

$$
\begin{gathered}
G_{\mu \nu}^{(l e a d)}=G_{\mu \nu}^{(c f t)}-\frac{2 \alpha^{\prime} \partial_{\mu} \phi \partial_{\nu} \phi}{1+\frac{1}{2} \alpha^{\prime} R}+\frac{2 \alpha^{\prime}(\partial \phi)^{2} G_{\mu \nu}}{1+\frac{1}{2} \alpha^{\prime} R}, \\
\phi^{(l e a d)}=\phi^{(c f t)}-\frac{1}{4} \ln \left(1+\frac{1}{2} \alpha^{\prime} R\right) .
\end{gathered}
$$

Note that the presence of the dilatonic terms $\alpha^{\prime} \partial_{\mu} \phi \partial_{\nu} \phi$ and $(\partial \phi)^{2} G_{\mu \nu}$ in (6.11) is consistent with (6.7), (6.9). However, since (6.11) and (6.9) were derived using specific properties of $D=2$ and $D=3$ backgrounds, they need not coincide in detail.28

As follows from the discussion in Section 5, (6.7) is also the transformation to the leading-order scheme for the $D=3 F$-model (see also Appendix C). This transformation must be universal: it should define the 'leading-order' scheme also for generic $D>3 F$ models. This is certainly true for the models (2.34) and is consistent with what we have found in Section 2. The background (2.28) corresponds to the solution in the 'leading-order' scheme

$$
G_{u v}^{(l e a d)}=B_{u v}^{(l e a d)}=\frac{1}{2} F, \quad G_{i j}^{(l e a d)}=\delta_{i j}, \quad \phi^{(l e a d)}=\phi^{\prime}+\frac{1}{2} \ln F, \quad \phi^{\prime} \equiv \phi_{0}+b_{i} x^{i}
$$

We thus conclude that the result of the path integral argument of Section 2 is consistent with the perturbative analysis of the solutions corresponding to (6.5) (cf. (2.26), (6.7)).

\section{Discussion}

We have considered two new classes of exact solutions to bosonic string theory. These take the form of the $F$-model (2.1) and the $K$-model (2.2) which are related by a leading order duality transformation. One can view these solutions as two different ways of extending a known spatial $D-2$ - dimensional euclidean CFT to obtain a $D$-dimensional lorentzian one. The $K$-model provides an interesting union of the standard gauged WZW and plane wave constructions. We have discussed a four and five dimensional example in Section 3 but clearly higher dimensional solutions can be constructed in an analogous

28 Since the CFT scheme is defined only for specific gauged WZW backgrounds, there may not exist a universal relation (valid in any $D$ ) between the CFT and the standard schemes. 
manner. Since these two classes of exact solutions are related by leading order duality, it appears likely that given any exact solution to string theory with a continuous symmetry, the solution obtained by a leading order duality transformation is also exact in some scheme.

Perhaps the most interesting solution in the class of $F$-models is the fundamental string (1.3). This solution has a curvature singularity at $r=0$. Furthermore, the effective string coupling $\exp \phi=g_{0} \sqrt{F}$ goes to zero at the singularity. The FS is the first example of an exact solution with these properties.29 It thus appears that this singularity might survive not only $\alpha^{\prime}$ - but also quantum string corrections. To establish this, two further results are needed. One must study the CFT corresponding to this classical solution and determine whether there is a singularity in a sense appropriate to string theory. The diverging curvature of the metric 'seen' by point-like string states by itself is not sufficient to ensure the existence of string singularities. One must also study the string loop corrections in some detail. The perturbative corrections are powers of the string coupling times some (nonlocal) functionals of the metric and dilaton which may still diverge at $r=0$ producing a large quantum correction. However, one should note that the string coupling vanishes faster in higher dimensions while the curvature diverges like $1 / r^{2}$ for all $D$. Thus it is possible that perturbative quantum effects are important only in low dimensions. In addition, there may be non-perturbative corrections, but they are likely to be small in the limit of small string coupling.

There are several arguments one might give to try to support the idea that the singularity in the fundamental string solution should be innocuous in string theory. First, the behavior of classical test strings in this background has been studied [43] and it was shown that test strings parallel to the source string and oriented in the same direction do not feel any force in the limit of small velocities.30 This same conclusion holds for all $F$-models. Second, quantum test strings have been studied in a shock wave background which has a singularity similar to the FS for $u=0$, but is flat elsewhere [45]. It was argued that string propagation remains well behaved. Third, strings may be dual to five-branes, and if one rescales the FS metric by a power of the dilaton to obtain the geometry seen by a five brane,

29 If one periodically identifies the direction along the string, the effective coupling is $\exp \left(\frac{1}{2} \varphi\right)$, $\varphi=2 \phi-\frac{1}{2} \ln G$, which is invariant under duality. Since the dual of the FS is a plane wave which is known to be exact, this provides a second (equivalent) example of a solution with these properties.

30 This is modified if we make a periodic identification to obtain strings of finite length $R$, and take the limit of small velocity $v$ holding $R v$ constant [44]. 
it does not have a curvature singularity [46] (although the dilaton still diverges). Finally, as we have said, the FS can be viewed as the field outside a straight fundamental string. Using the linearity of the equation for $F^{-1}$ one can consider the multi-string solution and study string scattering. Preliminary calculations show that this scattering is in agreement with the standard results of string scattering in flat spacetime [43,44]. This suggests that the FS solution is in some sense equivalent to the usual strings in string theory which are certainly non-singular objects!

While it may be true that the singularity in the FS is not serious, the above arguments are far from conclusive. In the first case, generic classical string configurations certainly do feel a force, and one must consider all states of a (quantum) test string before a singularity is declared harmless. In the next argument, the fact that the spacetime is flat away from $u=0$ means that a string will feel the singularity for at most an instant. For the FS, the singularity is present for all time. The third argument is relevant only if one wants to define a singularity in terms of the behavior of objects other than test strings. It is not clear whether this is a useful thing to do. Finally, the scattering calculations have so far been compared only at large impact parameter where the strong curvature regions do not play a significant role. More importantly, we have been viewing the FS as a particular nontrivial classical solution. The question of whether it is singular is not directly related to the scattering of two quantum strings. In particular, the fact that the string coupling goes to zero in the FS solution has no analog in the usual string scattering calculations in flat spacetime.

There is one unusual feature of the FS solution which is evident even at the level of the leading order string equations. While the FS is certainly a solution to these equations for $r \neq 0$, it is not a solution at the singularity due to the presence of $\delta$-function source terms. In general relativity, one never asks if the field equations hold at a singularity since in general this not a well defined question. However if string theory is indeed a 'theory of everything', one should presumably not add external sources. One might thus argue that the FS should not be viewed as an allowed classical background. The difficulty with this argument is that if one demands that the field equations hold everywhere, one is in danger of simply defining away the problem of singularities. Physically, one must study dynamical collapse situations to see whether the field equations break down, or (in some sense) remain satisfied for all time.

The key property of the $F$-models used in this paper is their chiral structure (the balance of the metric and antisymmetric tensor components) in the $(u, v)$ sector. That 
is why the path integral over $(u, v)$ can be computed exactly and one can prove their all-order conformal invariance. The fact that the integral over $(u, v)$ produces a local effective theory for the transverse coordinates $x^{i}$ is quite remarkable. That means that for the $F$-model backgrounds one has a formal $D \rightarrow D-2$ 'dimensional reduction': the correlators of operators which depend only on transverse coordinates 31 are exactly given by the correlators in the 'transverse' euclidean CFT. For example, for the $D=4 F$ models the 'transverse' CFT is two-dimensional, i.e. it is either the 'flat space with linear dilaton' (for the FS or the models (2.34)) or the $S L(2, R) / U(1)$ two dimensional black hole (for the solution constructed in Section 3 (3.17)). This is to be compared with, e.g., the Schwarzschild background where integrating out any pair of coordinates produces a complicated non-local two-dimensional effective theory.

We have seen in Section 5 that the $D=3 F$-model can be obtained as a gauged WZW model. It was previously shown that $F$-models of the form (2.34) can also be obtained as gauged WZW models. An open question is whether other $F$ - and $K$-models, in particular, the FS one, are also related to gauged WZW theories. It is known that some of the plane wave solutions admit a coset CFT interpretation [47. However, these are all of the special form (2.3) and thus are the simplest type of $K$-model . It is not clear whether this correspondence extends to the more general $K$-models considered here.

Another interesting question concerns supersymmetric versions of the $F$ - and $K$ models, and related superstring and heterotic string solutions. In particular, we can construct a $D=4$ heterotic string solution by adding the $(u, v)$-terms to the two dimensional 'monopole theory' constructed in [48]. This is essentially a reinterpretation (in a Kaluza-Klein or heterotic string manner) of the $(u, v) \times S U(2) D=5$ bosonic solution (3.2) as a $D=4$ heterotic one.

\section{Acknowledgements}

We wish to thank J. Gauntlett and K. Sfetsos for comments. G.H. was supported in part by NSF Grant PHY-9008502 and by EPSRC grant GR/J82041. A.A.T. acknowledges the support of PPARC.

31 Unfortunately, most of these operators do not describe physical states since a timelike momentum necessarily involves $u$ and $v$. 


\section{Appendix A. Leading order equations for the $F$-model}

We consider here the $F$-model with flat transverse space (1.1). Since

$$
G_{u v}=\frac{1}{2} F, \quad G_{i j}=\delta_{i j}, \quad B_{u v}=\frac{1}{2} F, \quad B_{i j}=0, \quad F \equiv \mathrm{e}^{2 h},
$$

the components of the Christoffel symbols are

$$
\Gamma_{u v}^{i}=-\frac{1}{2} F \partial_{i} h, \quad \Gamma_{v i}^{v}=\partial_{i} h, \quad \Gamma_{u i}^{u}=\partial_{i} h .
$$

The only non-trivial components of the curvature are (others reduce to them or vanish)

$$
R_{i u j}^{u}=-\partial_{j} \Gamma_{u i}^{u}-\Gamma_{u i}^{u} \Gamma_{u j}^{u}=-\partial_{i} \partial_{j} h-\partial_{i} h \partial_{j} h, \quad R_{u u v}^{u}=-\frac{1}{2} F \partial_{i} h \partial^{i} h .
$$

The Ricci tensor is then

$$
\begin{gathered}
R_{i j}=-2\left(\partial_{i} \partial_{j} h+\partial_{i} h \partial_{j} h\right), \quad R_{u v}=-\frac{1}{2} F\left(\partial^{2} h+2 \partial_{i} h \partial^{i} h\right), \\
R=-4 \partial^{2} h-6 \partial_{i} h \partial^{i} h .
\end{gathered}
$$

In addition, defining $H_{\mu \nu}^{2} \equiv H_{\mu \lambda \sigma} H_{\nu}^{\lambda \sigma}$, one has

$$
\begin{gathered}
H_{i u v}=F \partial_{i} h, \quad H_{u v}^{2}=-4 F \partial_{i} h \partial^{i} h, \quad H_{i j}^{2}=-8 \partial_{i} h \partial_{j} h, \\
\nabla_{i} \nabla_{j} \phi=\partial_{i} \partial_{j} \phi, \quad \nabla_{u} \nabla_{v} \phi=\frac{1}{2} F \partial_{i} h \partial^{i} \phi .
\end{gathered}
$$

The one-loop conformal invariance conditions can be obtained by extremizing the action (4.1). Varying with respect to $G_{\mu \nu}, B_{\mu \nu}$, and $\phi$ yields

$$
\begin{gathered}
R_{\mu \nu}-\frac{1}{4} H_{\mu \nu}^{2}+2 \nabla_{\mu} \nabla_{\nu} \phi=0 \\
\nabla^{\mu}\left(e^{-2 \phi} H_{\mu \nu \rho}\right)=0 \\
4 \nabla^{2} \phi-4(\nabla \phi)^{2}+R-\frac{1}{12} H_{\mu \nu \lambda} H^{\mu \nu \lambda}-\frac{2(D-26)}{3 \alpha^{\prime}}=0 .
\end{gathered}
$$

Eq. (A.8) yields

$$
(i j): \quad-\partial_{i} \partial_{j} h+\partial_{i} \partial_{j} \phi=0, \quad(u v): \quad-\frac{1}{2} \partial^{2} h+\partial_{i} h \partial^{i} \phi=0 .
$$

Eq. (A.9) does not produce any further independent conditions. From the first equation in (A.11) one has $\phi-h=\phi_{0}+b_{i} x^{i}$ where $b_{i}$ is a constant vector. The second equation 
in (A.11) can then be written $\partial^{2} F^{-1}=2 b^{i} \partial_{i} F^{-1}$, and the dilaton equation (A.10) implies $b_{i} b^{i}=-(D-26) / 6 \alpha^{\prime}$.

One might expect the curvature to be simpler using the connection with torsion

$$
\hat{\Gamma}_{\mu \nu}^{\lambda}=\Gamma_{\mu \nu}^{\lambda}+\frac{1}{2} H_{\mu \nu}^{\lambda}
$$

Then

$$
\hat{\Gamma}_{u v}^{i}=0, \quad \hat{\Gamma}_{v u}^{i}=-F \partial_{i} h, \quad \hat{\Gamma}_{i v}^{v}=0, \quad \hat{\Gamma}_{v i}^{v}=2 \partial_{i} h, \quad \hat{\Gamma}_{u i}^{u}=0, \quad \hat{\Gamma}_{i u}^{u}=2 \partial_{i} h .
$$

The curvature for $\hat{\Gamma}_{\mu \nu}^{\lambda}$ is the following

$$
\hat{R}_{i u j}^{u}=-2 \partial_{i} \partial_{j} h, \quad \hat{R}_{i v j}=0, \quad \hat{R}_{u j v}^{i}=0, \quad \hat{R}_{v j u}^{i}=-F \partial^{i} \partial_{j} h, \quad \hat{R}_{u u v}^{u}=0 .
$$

It vanishes when $h$ is a linear function of $x$, e.g. for the $S L(2, R)$ WZW model $\left(F=e^{-2 b x}\right)$ as it should since this is the group space case. To obtain a vanishing curvature for the general $F$-model we would need to 'add' dilatonic terms to $\partial_{i} \partial_{j} h$ and $\partial^{i} \partial_{j} h$ terms in (A.14). It is not clear if this can be done in a systematic way by modifying the connection.

\section{Appendix B. A generalization of the $F$-model}

In this appendix we point out that there is a slight generalization of the $F$-model which can also be shown to be conformal using the arguments of Section 2. This is motivated by the following generalization of the $K$-model (see e.g. [2, [2]). When the transverse space is flat one can extend the $K$-model by introducing an antisymmetric tensor background of the form $B_{i u}=B_{i}(x)$. The $K$-model then becomes

$$
L_{K}=\partial u \bar{\partial} v+K(x) \partial u \bar{\partial} u+B_{i}(x)\left(\partial x^{i} \bar{\partial} u-\partial u \bar{\partial} x^{i}\right)+\partial x^{i} \bar{\partial} x_{i}+\alpha^{\prime} \mathcal{R}\left(a+b_{i} x^{i}\right)
$$

In this case, $H_{\mu \nu \lambda}$ is again proportional to the covariantly constant null vector and one can

show that all terms in the conformal invariance equations for the $K$-model which involve more than two powers of $H$ vanish identically. If we define $H_{i j} \equiv 2 \partial_{[i} B_{j]}$, these equations become (cf. (2.20))

$$
\begin{gathered}
\partial^{j}\left(e^{-2 \phi} H_{i j}\right)=0 \\
-\frac{1}{2} \partial^{2} K+b^{i} \partial_{i} K-\frac{1}{4} H^{i j} H_{i j}+2 \partial_{u}^{2} \phi+O\left(\alpha^{\prime s}\left(\partial^{s} H\right)^{2}\right)=0 .
\end{gathered}
$$


The last equation equation still includes $\alpha^{\prime}$ corrections but these vanish in the simple case of a 'constant field strength'

$$
b_{i}=0, \quad B_{i}=-\frac{1}{2} H_{i j} x^{j}, \quad H_{i j}=\mathrm{const}, \quad-\frac{1}{2} \partial^{2} K-\frac{1}{4} H^{i j} H_{i j}+2 \partial_{u}^{2} \phi=0 .
$$

When $K$ and $\phi$ are both independent of $u$, one can construct a generalized $F$-model which is dual to this solution. Our conjecture in Section 1 implies that this generalized $F$-model should also be an exact solution. We now show that this is indeed the case.

The Lagrangian $u$-dual to the generalized $K$-model (B.1) is

$$
\begin{gathered}
L_{F}=F(x)\left(\partial u+B_{i} \partial x^{i}\right)\left(\bar{\partial} v+B_{i} \bar{\partial} x^{i}\right)+\partial x^{i} \bar{\partial} x_{i} \\
+\alpha^{\prime} \mathcal{R}\left(\phi_{0}+b_{i} x^{i}+\frac{1}{2} \ln F\right), \quad F=K^{-1} .
\end{gathered}
$$

This generalized $F$-model is still chiral in the $u, v$ directions. Integrating over $v$ and $u$ we find the following expression for the generating functional (cf. (2.22))

$$
\begin{gathered}
\exp (-W[U, V, X, \gamma])=Z_{0}(\gamma) \int[d x] \exp \left(-\frac{1}{\pi \alpha^{\prime}} \int d^{2} z\left[G_{i j}^{\prime} \partial x^{i} \bar{\partial} x^{j}\right.\right. \\
\left.\left.+B_{i}(x)\left(\partial U^{\prime} \bar{\partial} x^{i}-\partial x^{i} \bar{\partial} V\right)-F^{-1}(x) \partial U^{\prime} \bar{\partial} V+\alpha^{\prime} \mathcal{R}\left(\phi_{0}+b_{i} x^{i}\right)+X \partial \bar{\partial} x\right]\right),
\end{gathered}
$$

where $G_{i j}^{\prime}$ and $T$ are the same as in (2.15) and (2.23). By power counting the conformal invariance conditions for the couplings in $W$ must be at most quadratic in $B_{i}$. We thus get back to the conditions $(\overline{B .2})$. In the leading-order scheme we thus obtain the following 'constant field strength' solution

$$
b_{i}=0, \quad B_{i}=-\frac{1}{2} H_{i j} x^{j}, \quad H_{i j}=\mathrm{const}, \quad \partial^{2} F^{-1}=-\frac{1}{2} H^{i j} H_{i j} .
$$

The solution that generalizes the FS one (1.3) is thus

$$
F^{-1}=1-\frac{H^{i j} H_{i j}}{4(D-2)} r^{2}+\frac{M}{r^{D-4}}, \quad D>4 .
$$

\section{Appendix C. Shifts of metric and dilaton in $D=3 F$-model}

To clarify the meaning of the shifts of the metric and dilaton implied by the path integral argument of Section 2, let us consider first a particular example of a $D=3 F$ model - the $S L(2, R)$ WZW model (5.6): $F=e^{-2 b x}, \phi=\phi_{0}, \alpha^{\prime} b^{2}=1 / k$. Using the method of section 2 one can demonstrate explicitly its all-order conformal invariance and 
compute the exact value of its central charge.32 In fact, integrating over $u$ and $v$ we find according to (2.15) the following $x$-model:

$$
\begin{gathered}
L=G_{x x}(x) \partial x \bar{\partial} x+\alpha^{\prime} \mathcal{R} \phi, \quad \phi=\phi_{0}-\frac{1}{2} \ln F=\phi_{0}+b x, \\
G_{x x}=1-\frac{1}{2} \alpha^{\prime}\left(\partial_{x} \ln F\right)^{2}=1-2 \alpha^{\prime} b^{2}=\frac{k-2}{k} .
\end{gathered}
$$

The integral over $(u, v)$ thus produces the effective renormalisation $k \rightarrow k-2$ of the coefficient of the $\partial x \bar{\partial} x$ term. We have assumed that the measure factor $F_{0}$ in $(2.12)$ is equal to $F=e^{-2 b x}$ (as implied by the Haar measure). The conformal invariance condition (2.24) is then satisfied automatically and the corresponding central charge condition becomes (cf. (3.8))

$$
0=D-26+6 \alpha^{\prime} G^{x x} \partial_{x} \phi \partial_{x} \phi=D-26+\frac{6 \alpha^{\prime} b^{2}}{1-2 \alpha^{\prime} b^{2}}=-26+\frac{3 k}{k-2} .
$$

This calculation was done in the leading-order scheme where the original background fields do not receive $\alpha^{\prime}$ corrections. Alternatively, we may start with the corrected metric in (2.25) and after having integrated out $u, v$ get just $G_{x x}=1$. In that scheme the central charge equation is thus

$$
0=D-26+6 \alpha^{\prime} G^{x x} \partial_{x} \phi \partial_{x} \phi=D-26+6 \alpha^{\prime} b^{2}
$$

To obtain the same exact expression for the central charge we need to start with the WZW action in the CFT scheme (i.e. with $k \rightarrow k-2)$ so that $\alpha^{\prime} b^{2}$ is identified with $1 /(k-2)$ (see also [10,11]).

Let us now repeat this argument for the general $D=3 F$-model, i.e. the gauged WZW model (5.11). Changing the variables to $w, \tilde{w}: A=\partial w, \bar{A}=\bar{\partial} \tilde{w}$ we have in the $y=0$ gauge

$$
L_{g w z w}=k\left[b^{2} \partial x \bar{\partial} x+\mathrm{e}^{-2 b x}(\partial u+\lambda \partial w)(\bar{\partial} v+\nu \bar{\partial} \tilde{w})+\rho^{2} \partial w \bar{\partial} \tilde{w}\right] .
$$

This is the expression in the leading-order scheme for the WZW theory. In the CFT scheme $k \rightarrow k-2, \rho^{2} \rightarrow \rho^{2} \frac{k}{k-2}$. As was already noted above, this transformation is trivial since it can be 'undone' by rescaling of the coordinates. If we first change the coordinates $u \rightarrow u^{\prime}-\lambda w, v \rightarrow v^{\prime}-\nu \tilde{w}$ then (C.4) becomes the sum of decoupled actions for the

32 A similar computation of the central charge for WZW models admitting the Gauss decomposition parametrisation was discussed in 49 (see also [13]). 
$S L(2, R)$ WZW model and the free $R \times R$ model for $(w, \tilde{w})$. The theory is thus obviously conformally invariant. Integrating over $u^{\prime}, v^{\prime}$ and $w, \tilde{w}$ we get the same resulting $x$-theory (C.1), (C.2) as in the ungauged WZW case. The integral over $w, \tilde{w}$ gives only a constant contribution if we assume that the measure factor $F_{0 w}$ corresponding to $(w, \tilde{w})$ is trivial.

Equivalent result should be found if we first integrate over $A, \bar{A}$ or $w, \tilde{w}$ (as we are supposed to do in order to obtain a $\sigma$-model corresponding to a gauged WZW model). We can compute the resulting effective action by applying (2.10), (2.12) to the integral over $w, \tilde{w}$ with the action $\sim \int d^{2} z F_{w}(x) \partial w \bar{\partial} \tilde{w}$,

$$
F_{w} \equiv 1+a \mathrm{e}^{-2 b x}, \quad a=\lambda \nu / \rho^{2} .
$$

Taking the corresponding measure factor to be equal to $F_{w}$ we find (cf. (5.3), (5.12))

$$
\begin{gathered}
L_{F}=F \partial u \bar{\partial} v+\left(1-\frac{1}{2} \alpha^{\prime} \partial \ln F_{w} \partial \ln F_{w}\right) \partial x \bar{\partial} x+\alpha^{\prime} \mathcal{R}\left(\phi_{0}-\frac{1}{2} \ln F_{w}\right), \\
F^{-1}=a+\mathrm{e}^{2 b x}=\mathrm{e}^{2 b x} F_{w} .
\end{gathered}
$$

If we now integrate over $u, v$ (assuming that the measure factor for $(u, v)$ is $F_{0}=F$ as would be natural if we would have started with a $\sigma$-model (C.6) with the path integral measure defined by the corresponding $\sigma$-model metric) we get the $x$-theory with (cf. (C.1))

$$
\begin{gathered}
G_{x x}^{\prime}=1-\frac{1}{2} \alpha^{\prime} \partial \ln F_{w} \partial \ln F_{w}-\frac{1}{2} \alpha^{\prime} \partial \ln F \partial \ln F, \\
\phi=\phi_{0}-\frac{1}{2} \ln F^{\prime}-\frac{1}{2} \ln F=\phi_{0}+b x .
\end{gathered}
$$

While the dilaton (C.8) is the same as in (C.1) the $\alpha^{\prime}$-term in the metric does not reduce to the expected result

$$
G_{x x}^{\prime}=1-\frac{1}{2} \alpha^{\prime} \partial\left(\ln F_{w}+\ln F\right) \partial\left(\ln F_{w}+\ln F\right)=1-2 \alpha^{\prime} b^{2} .
$$

The reason for this paradox lies in the fact that the redefinitions of $(u, v)$ should be consistent with covariance properties of the measure, i.e. they do not, in general, preserve the covariance of the theory. As in the path integral argument in Section 2, extra local counterterms are to be added to get a consistent result. In the present case we need to add a local counterterm leading to the 'mixing' term $-\alpha^{\prime} \partial \ln F \partial \ln F_{w}$ in $G_{x x}$ in (C.7). Then the final result (C.9) is the same as in the manifestly 'conformal' approach when one first redefines $u, v$ to decouple them from $w, \tilde{w}$. 
More generally, one can consider the following analog of the $(u, v, w, \tilde{w})$ part of (C.4):

$$
L=F_{1}(\partial u+\partial w)(\bar{\partial} v+\bar{\partial} \tilde{w})+F_{2} \partial w \bar{\partial} \tilde{w} \equiv F_{a b} \partial u^{a} \bar{\partial} v^{b}
$$

The two ways of computing this integral (by first integrating over $u, v$ and then over $w, \tilde{w}$ or vice versa) give equivalent results, i.e. the relation 33

$$
\Delta I=\Delta I_{1}\left(F_{1}\right)+\Delta I_{2}\left(F_{2}\right)=\Delta I_{1}\left(\frac{F_{1} F_{2}}{F_{1}+F_{2}}\right)+\Delta I_{2}\left(F_{1}+F_{2}\right),
$$

is true only if the measure on the full $(u, v, w, \tilde{w})$ space is consistently assumed to be the same in both cases.

$33 \Delta I_{1}$ and $\Delta I_{2}$ correspond to the $(u, v)$ and $(w, \tilde{w})$ integrals and are the same as in 2.12). 


\section{References}

[1] R. Güven, Phys. Lett. B191 (1987) 275; D. Amati and C. Klimčík, Phys. Lett. B219 (1989) 443; R. Rudd, Nucl. Phys. B352 (1991) 489.

[2] G. Horowitz and A. Steif, Phys. Rev. Lett. 64 (1990) 260; Phys. Rev. D42 (1990) 1950; G. Horowitz, in: Strings '90, (eds. R Arnowitt et. al.) World Scientific (1991).

[3] H. de Vega and N. Sanchez, Phys. Rev. D45 (1992) 2783; Class. Quantum Grav. 10 (1993) 2007.

[4] A. Tseytlin, Nucl. Phys. B390 (1993) 153.

[5] E. Witten, Phys. Rev. D44 (1991) 314.

[6] I. Bars and K. Sfetsos, Mod. Phys. Lett. A7 (1992) 1091; P. Horava, Phys. Lett. B278 (1992) 101; P. Ginsparg and F. Quevedo, Nucl. Phys. B385 (1992) 527.

[7] R. Dijkgraaf, H. Verlinde and E. Verlinde, Nucl. Phys. B371 (1992) 269.

[8] I. Bars and K. Sfetsos, Phys. Rev. D46 (1992) 4510; Phys. Rev. D48 (1993) 844.

[9] A. Tseytlin, Nucl. Phys. B399 (1993) 601; Nucl. Phys. B411 (1994) 509.

[10] A. Tseytlin, Phys. Lett. B317 (1993) 559.

[11] K. Sfetsos and A. Tseytlin, Phys. Rev. D49 (1994) 2933.

[12] A. Giveon and E. Kiritsis, Nucl. Phys. B411 (1994) 487.

[13] C. Klimčík and A. Tseytlin, "Exact four dimensional string solutions and Toda-like sigma models from null-gauged WZNW models", preprint Imperial/TP/93-94/17, hep-th/9402120.

[14] A. Dabholkar, G. Gibbons, J. Harvey, and F. Ruiz, Nucl. Phys. B340 (1990) 33.

[15] E. Bergshoeff, I. Entrop, and R. Kallosh, "Exact Duality in String Effective Action", SU-ITP-93-37; hep-th/9401025.

[16] G. Horowitz and A. Strominger, Nucl. Phys. B360 (1991) 197.

[17] J. Horne, G. Horowitz, and A. Steif, Phys. Rev. Lett. 68 (1992) 568; G. Horowitz, in: String Theory and Quantum Gravity '92, (eds. J. Harvey, R. Iengo, K. Narain, S. Randjbar-Daemi, and H. Verlinde) World Scientific (1993).

[18] J. Horne and G. Horowitz, Nucl. Phys. B368 (1992) 444.

[19] T. Buscher, Phys. Lett. B194 (1987) 59; Phys. Lett. B201 (1988) 466.

[20] A. Tseytlin, Mod. Phys. Lett. A6 (1991) 1721.

[21] G. Horowitz and D. Welch, Phys. Rev. Lett. 71 (1993) 328; N. Kaloper, Phys. Rev. D48 (1993) 2598.

[22] S. Elitzur, A. Forge and E. Rabinovici, Nucl. Phys. B359 (1991) 581; G. Mandal, A. Sengupta and S. Wadia, Mod. Phys. Lett. A6 (1991) 1685.

[23] D. Garfinkle, Phys. Rev. D46 (1992) 4286.

[24] A. Schwarz and A. Tseytlin, Nucl. Phys. B399 (1993) 691.

[25] R. Kallosh and A. Morozov, Int. J. Mod. Phys. A3 (1988) 1943. 
[26] P. Mansfield and J. Miramontes, Phys. Lett. B199 (1988) 224; A. Tseytlin, Phys. Lett. B208 (1988) 228; Phys. Lett. B223 (1989) 165.

[27] C. Callan and Z. Gan, Nucl. Phys. B272 (1986) 647; A. Tseytlin, Phys. Lett. B178 (1986) 34.

[28] S. Mukhi, Phys. Lett. B162 (1985) 345; S. De Alwis, Phys. Lett. B164 (1985) 67.

[29] C. Hull and P. Townsend, Nucl. Phys. B274 (1986) 349.

[30] I. Jack, D. Jones and J. Panvel, Nucl. Phys. B393 (1993) 95.

[31] J. Scherk, Nucl. Phys. B31 (1971) 222; J. Scherk and J. Schwarz, Nucl. Phys. B81 (1974) 118; T. Yoneya, Progr. Theor. Phys. 51 (1974) 1907.

[32] E. Fradkin and A. Tseytlin, Phys. Lett. B158 (1985) 316; Nucl. Phys. B261 (1985) 1.

[33] C. Lovelace, Nucl. Phys. B273 (1986) 413.

[34] C. Callan, D. Friedan, E. Martinec and M. Perry, Nucl. Phys. B262 (1985) 593.

[35] A. Tseytlin, Phys. Lett. B176 (1986) 92; Nucl. Phys. B276 (1986) 391.

[36] A. Tseytlin, Phys. Lett. B208 (1988) 221.

[37] R. Metsaev and A. Tseytlin, Nucl. Phys. B293 (1987) 385.

[38] I. Jack and D. Jones, Phys. Lett. B200 (1988) 453.

[39] R. Metsaev and A. Tseytlin, Phys. Lett. B185 (1987) 52.

[40] V. Fateev, E. Onofri and Al. Zamolodchikov, Nucl. Phys. B406 (1993) 521.

[41] T. Banks, M. Dine, H. Dijkstra and W. Fischler, Phys. Lett. B212 (1988) 45.

[42] K. Sfetsos, Nucl. Phys. B389 (1993) 424.

[43] C. Callan and R. Khuri, Phys. Lett. B261 (1991) 363; R. Khuri, Nucl. Phys. B403 (1993) 335.

[44] J. Gauntlett, J. Harvey, M. Robinson, and D. Waldram, Nucl. Phys. B411 (1994) 461.

[45] H. de Vega and N. Sanchez, Phys. Lett. B244 (1990) 215.

[46] M. Duff, G. Gibbons and P. Townsend, "Macroscopic superstrings as interpolating solitons", DAMTP/R-93/5, hep-th/9405124.

[47] C. Nappi and E. Witten, Phys. Rev. Lett. 71 (1993) 3751; E. Kiritsis and C. Kounnas, Phys. Lett. B320 (1994) 264; D. Olive, E. Rabinovici and A. Schwimmer, Phys. Lett. B321 (1994) 361; K. Sfetsos, Phys. Lett. B324 (1994) 335; "Gauged WZW models and Non-abelian Duality", THU-94/01, hep-th/9402031; I. Antoniadis and N. Obers, "Plane Gravitational Waves in String Theory", CPTH-A299.0494, hepth/9403191; K. Sfetsos and A. Tseytlin, "Four Dimensional Plane Wave String Solutions with Coset CFT Description", preprint THU-94/08, hep-th/9404063.

[48] S. Giddings, J. Polchinski and A. Strominger, Phys. Rev. D48 (1993) 5784.

[49] A. Gerasimov, A. Morozov, M. Olshanetsky, A. Marshakov and S. Shatashvili, Int. J. Mod. Phys. A5 (1990) 2495. 